\title{
From a discrete to a continuous model for interpulse interference with a frequency-comb laser
}

\section{Citation for published version (APA):}

Zeitouny, M. G., Cui, M., Bhattacharya, N., Urbach, H. P., Berg, S. A., \& Janssen, A. J. E. M. (2010). From a discrete to a continuous model for interpulse interference with a frequency-comb laser. Physical Review A : Atomic, Molecular and Optical Physics, 82(2), 023808-1/5. [023808]. https://doi.org/10.1103/PhysRevA.82.023808

DOI:

10.1103/PhysRevA.82.023808

Document status and date:

Published: 01/01/2010

\section{Document Version:}

Publisher's PDF, also known as Version of Record (includes final page, issue and volume numbers)

\section{Please check the document version of this publication:}

- A submitted manuscript is the version of the article upon submission and before peer-review. There can be important differences between the submitted version and the official published version of record. People interested in the research are advised to contact the author for the final version of the publication, or visit the $\mathrm{DOI}$ to the publisher's website.

- The final author version and the galley proof are versions of the publication after peer review.

- The final published version features the final layout of the paper including the volume, issue and page numbers.

Link to publication

\section{General rights}

Copyright and moral rights for the publications made accessible in the public portal are retained by the authors and/or other copyright owners and it is a condition of accessing publications that users recognise and abide by the legal requirements associated with these rights.

- Users may download and print one copy of any publication from the public portal for the purpose of private study or research.

- You may not further distribute the material or use it for any profit-making activity or commercial gain

- You may freely distribute the URL identifying the publication in the public portal.

If the publication is distributed under the terms of Article 25fa of the Dutch Copyright Act, indicated by the "Taverne" license above, please follow below link for the End User Agreement:

www.tue.nl/taverne

Take down policy

If you believe that this document breaches copyright please contact us at:

openaccess@tue.nl

providing details and we will investigate your claim. 


\title{
From a discrete to a continuous model for interpulse interference with a frequency-comb laser
}

\author{
M. G. Zeitouny, ${ }^{*}$ M. Cui, N. Bhattacharya, and H. P. Urbach \\ Technische Universiteit Delft, Lorentzweg 1, 2628 CJ Delft, The Netherlands \\ S. A. van den Berg \\ VSL, Thijsseweg 11, 2629 JA Delft, The Netherlands \\ A. J. E. M. Janssen \\ Technical University Eindhoven, Department EE and EURANDOM, LG-1, P. O. Box 513, 5600 MB Eindhoven, The Netherlands
}

(Received 1 April 2010; revised manuscript received 8 June 2010; published 17 August 2010)

\begin{abstract}
We have investigated correlation patterns generated by a frequency-comb laser in a dispersive unbalanced Michelson interferometer and apply the developed formalism to the case of distance metrology. Due to group velocity dispersion, the position of the brightest fringe of the correlation pattern, which is used for distance determination, cannot be derived by simply using the definition of group refractive index of the dispersive medium. It is shown that the discrete spectrum of the optical frequency comb gives rise to correlation functions which can be represented by a series, namely a discrete correlation series. We have developed a general formalism, valid for any pulse train, extending the discrete model to a continuous model of cross-correlation functions using the Poisson summation. Our model is relevant for any offset and repetition frequency of the frequency comb. From the continuous cross-correlation model we show that, even for a homogeneous dispersive medium the position of the brightest fringe varies nonlinearly for small delay distances and stabilizes for longer ones. We have compared the theoretical results to measurements of pulse propagation in air for path-lengths up to $200 \mathrm{~m}$.

DOI: 10.1103/PhysRevA.82.023808

PACS number(s): 42.25.Hz, 42.25.Kb, 42.62.Eh
\end{abstract}

\section{INTRODUCTION}

Recent advances in the field of ultrashort pulse lasers have led to the development of reliable sources of carrierenvelope-phase stabilized femtosecond pulses [1-3]. The pulse train generated by such a source has a frequency spectrum that consists of discrete, regularly spaced lines known as a frequency comb. In this case both the frequency repetition and the carrier-envelope-offset (CEO) frequency, are referenced to a frequency standard, like an atomic clock. As a result the accuracy of the frequency standard is transferred to the optical domain, with the frequency comb as transfer oscillator. These unique properties allow the frequency comb to be applied as a versatile tool, not only for time and frequency metrology [4-7], but also in fundamental physics [8,9], high-precision spectroscopy [10-12], and laser noise characterization [13-15]. The pulse-to-pulse phase relationship of the light emitted by the frequency comb has opened up new directions for long range highly accurate distance measurement [16-23]. For nondispersive media an arbitrary plane wave pulse would propagate unaltered in shape at the phase velocity of the wave field in the medium. In that case, the analysis of the resulting interfering field or correlation patterns is straightforward. However, the analysis acquires complexity for pulse propagation in a dispersive medium like air [21,22].

Propagation of individual pulses in dispersive media has been extensively studied over the last decades. Most analysis of ultrashort pulses for interference [24] or propagation [25-27] is based on Gaussian pulse models. The electric fields of the Gaussian pulses generate symmetric interference patterns even if second-order dispersion is taken into account. However,

\footnotetext{
*M.Zeitouny@tudelft.nl
}

practical applications like distance measurement use laser pulses with asymmetric non-Gaussian spectra and therefore require a generalized approach.

In this paper, we present a study of the formation of correlation patterns in a dispersive unbalanced Michelson interferometer using a frequency-comb laser as a source. This study is specifically intended for distance measurement in dispersive media. For accurate distance determination, precise knowledge of the position of the brightest fringe, the fringe having maximum contrast, of the correlation pattern is required. We show the influence of the propagation of pulses in air on the position of the brightest fringe of the correlation pattern for nonsymmetric spectra. The position of maximum coherence is linked to the asymmetry of the spectrum and the environmental parameters. Theoretical and numerical analysis show the difference between the correlation patterns generated by symmetric spectra (such as a Gaussian spectrum) and arbitrary spectra emitted from a laser.

We approach the problem by representing the correlation function from the optical frequency comb by a discrete correlation series. Using the Poisson summation formula we extend the above formalism to a continuous model of cross-correlation functions. Our model is relevant for any offset and repetition frequency of the frequency comb. The model allows us to show that even for a homogenous dispersive medium the position of the brightest fringe varies nonlinearly for small delay distances and stabilizes for longer ones. The distance where nonlinear effects are important is shown to be dependent on the properties of the dispersive media and the initial spectrum of the transmitted pulse. In case of very large delay distances the particular values of the frequencies present in the spectrum play an important role since only specific frequencies contribute to specific fringes in the crosscorrelation. Therefore, the precise values of the offset and 
repetition frequencies become important and the model can be used to determine the properties of the cross-correlations in the asymptotic limit of large delay distances, as compared to a model where the offset frequency is ignored. This makes the present continuous model valid for a large range of delay distances.

The pulse-to-pulse phase stability of the frequency-comb source offers the opportunity, for the first time, to give a comparison between a theoretical and experimental analysis of the formation of temporal interference fringes in dispersive media. The theory and numerical simulation developed in this paper will be compared to measurements of path lengths up to $200 \mathrm{~m}$

This paper is organised as follows. In Sec. II, a theoretical model of the cross-correlation functions obtained from the discrete spectrum of the frequency comb has been developed followed by a comparison with some experimental measurements. In Sec. III, we extend the discrete formulation of the cross-correlation to a continuous model using the Poisson summation formula. This is followed in Sec. IV by a study of the position of the brightest fringe in the cross-correlation based on the continuous model and the quadratic phase approximation. In Sec. $\mathrm{V}$ we estimate the distance where nonlinear effects are dominant in a dispersive medium. Finally, the main conclusions of this work are summarized in Sec. VI.

\section{ANALYSIS OF PULSE CROSS-CORRELATION FUNCTIONS}

First, we give a short description of a generalized distance metrology setup using a Michelson interferometer. In 2004, a scheme for measuring long distances in space with a stabilized femtosecond frequency comb was proposed [16]. The scheme is based on a Michelson type interferometry with optical interference between individual pulses. The basic elements of this scheme are shown in Fig. 1. The pulse train from the laser is split into two beams which are recombined after having passed through various optical delays. The short arm is scanned over a fixed range using, among others, a piezoelectric-transducer, while the long arm is displaced over a distance that is to be determined. The intensity of the recombined beam is recorded as a function of the variable delay, i.e., piezo-element positions. In vacuum, a maximum coherence is obtained when the path length difference is $q l_{p p}$ between both arms, where $q$ is equal to an integer, and $l_{p p}$ is the effective laser cavity length. In vacuum $l_{p p}=c / f_{r}$ or the pulse-to-pulse distance, where $f_{r}$ is the pulse repetition of the laser and $c$ is the vacuum velocity of light. In dispersive media, one may expect that $l_{p p}=c /\left(n_{g} f_{r}\right)$, where $n_{g}$ denotes the group refractive index of the medium. The group refractive index $n_{g}$ and/or the corresponding group velocity of a pulse propagating in dispersive media has been the subject of extensive analysis [25-28]. Group velocity has been traditionally described as the rate at which the envelope of a group of waves travels through the dispersive medium. Propagation in a dispersive medium, however, leads to substantial broadening and shape deformation of the pulse due to group velocity dispersion [25]. For the case of an ultrashort pulse in a dispersive medium the classical definition of group velocity becomes questionable, in particular in the region of anomalous dispersion [26]. However, even in the case of quadratic dispersive media in the region of normal dispersion with negligible absorption, the use of group velocity for metrological purposes can be misleading. For the case of distance measurement, the pulse traveling in the long arm acquires a chirp which modifies the shape and width of correlation patterns extensively. The position of the maximum of the correlation differs considerably from $q l_{p p}$, where $l_{p p}$ is calculated using the group refractive index $n_{g}$ at the maximum of the source spectrum. The relation between the position of the maximum of a correlation pattern and this particular choice of $n_{g}$ will be discussed rigourously in the next section.

The complete mathematical and physical basis for the group velocity approximation in the context of a pulse propagating through quadratic dispersive media was laid by Jones [28] using the quasi-monochromatic or slowly-varying envelope approximation. An alternative treatment using $A B C D$ matrices was given by Dijaili et al. [27]. We extend the treatment of pulse propagation to the interference pattern formed by the overlap of the chirped pulse with a nonchirped one. Our treatment is mainly for pulses with a nonsymmetric spectrum propagating in a passive dispersive media like air in the regime of normal dispersion.

\section{A. Cross-correlations in dispersive media}

The field cross-correlation is readily measured experimentally by placing a slow detector at the output of the Michelson interferometer. The detector is illuminated by the input electric field $\mathcal{E}\left(x_{1}, t\right)$ coming from one arm, and by the delayed field $\mathcal{E}\left(x_{2}, t\right)$ from the other arm. If the time response of the detector is much larger than the time duration of the signal $\mathcal{E}(t)$, or if the recorded signal is integrated, the detector measures the

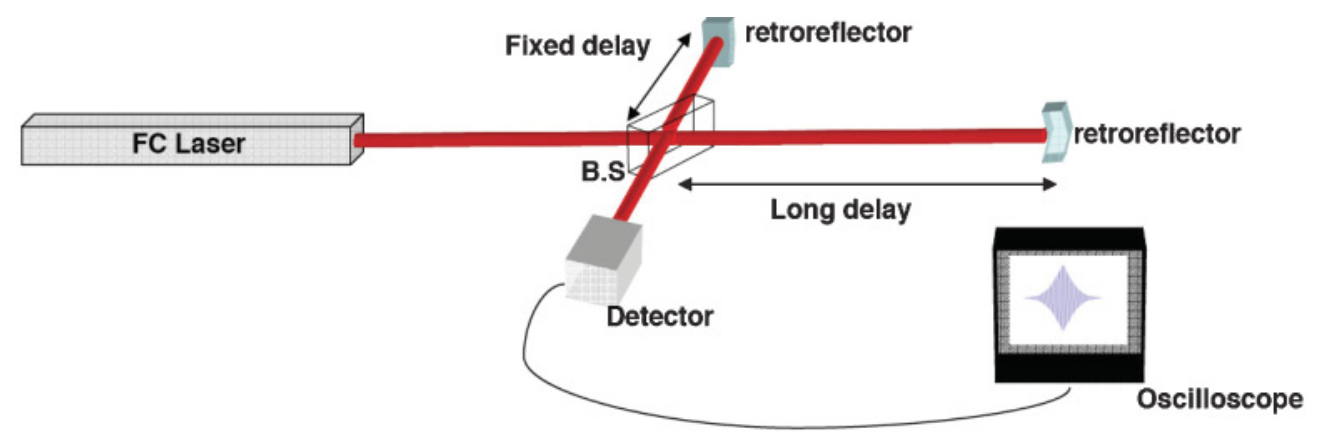

FIG. 1. (Color online) Schematic of the experimental setup. 
intensity. In this section we calculate the intensity detected by a slow detector as the interference of two electric fields which have traveled the distances $x_{1}$ and $x_{2}$, respectively.

The frequency spectrum emitted by a mode-locked laser consists of a comb of regularly spaced frequencies, $\omega_{m}=$ $m \omega_{r}+\omega_{0}$, where $\omega_{0}$ is the common offset frequency, $m$ is an positive integer, and $\omega_{r}$ is the repetition frequency $f_{r}$ expressed in angular notation, i.e., $\omega_{r}=2 \pi f_{r}=\frac{2 \pi}{T_{r}}$ and $T_{r}=\frac{1}{f_{r}}$. Here $T_{r}$ is the time interval between the pulses. The offset frequency $\omega_{0}$, is caused by the difference between the group velocity and the phase velocity inside the laser cavity. Both $\omega_{0}$ and $\omega_{r}$ are generally stabilized to an atomic clock.

In our experiments we use a Ti:sapphire laser. The central frequency of the comb is $\omega_{c}=2.3254 \times 10^{15} \mathrm{rad} / \mathrm{s}$, corresponding to a wavelength of $810 \mathrm{~nm}$ in vacuum, the bandwidth is typically $\Delta \omega \approx 5 \times 10^{14} \mathrm{rad} / \mathrm{s}$, which corresponds to a pulse width of $\Delta x \approx 12 \mu \mathrm{m}$ and a pulse duration of $40 \mathrm{fs}$. The frequency offset is $\omega_{0} \approx 113 \times 10^{7} \mathrm{rad} / \mathrm{s}$ and the repetition frequency is $\omega_{r}=6.28 \times 10^{9} \mathrm{rad} / \mathrm{s}$, corresponding to a cavity length $l_{p p}=30 \mathrm{~cm}$ and period $T_{r} \approx 1 \mathrm{~ns}$.

We now aim to obtain an expression for the crosscorrelation function from the laser source described above in presence of dispersive media. We use the Fourier transform as $(\mathcal{F} f)(\omega)=\int_{-\infty}^{\infty} f(t) \exp (-i \omega t) d t$ with the inversion formula $f(t)=\frac{1}{2 \pi} \int_{-\infty}^{\infty}(\mathcal{F} f)(\omega) \exp (i \omega t) d \omega$, and Parseval's formula $\int_{-\infty}^{\infty}(\mathcal{F} f)(\omega)(\mathcal{F} g)^{*}(\omega) d \omega=2 \pi \int_{-\infty}^{\infty} f(t) g^{*}(t) d t$

The field of the pulse emitted by the laser, propagating in the direction of positive $x$, at $x=0$ can be written as $\mathcal{E}(0, t)=\sum_{m=0}^{\infty} A_{m} \cos \left[\left(m \omega_{r}+\omega_{0}\right) t+\phi_{m}\right]$, where $A_{m}$ is a real amplitude and $\phi_{m}$ is the phase. In the calculations that follow it will be convenient to use negative frequencies.

We rewrite the field as $\mathcal{E}(0, t)=\operatorname{Re}\left[\exp \left(i \omega_{0} t\right) \mathcal{E}^{p}(0, t)\right]$ with $\mathcal{E}^{p}(0, t)=\sum_{m=0}^{\infty} a_{m} \exp \left(i m \omega_{r} t\right)$ where $a_{m}=A_{m} \exp \left(i \phi_{m}\right)$. Further we have $\mathcal{F}\left[\mathcal{E}^{p}(0, t)\right](\omega)=2 \pi \sum_{m=0}^{\infty} a_{m} \delta\left(\omega-m \omega_{r}\right)$. Therefore, the Fourier transform of the pulse emitted by the laser is $\mathcal{F}[\mathcal{E}(0, t)](\omega)=\pi \sum_{m=0}^{\infty} a_{m} \delta\left(\omega-m \omega_{r}-\omega_{0}\right)+$ $\pi \sum_{m=0}^{\infty} a_{m}^{*} \delta\left(\omega+m \omega_{r}+\omega_{0}\right)$.

Next we consider the propagation of the pulse in a dispersive medium with refractive index $n(\omega)$ and assuming that the pulse propagates in the direction of positive $x$ we can write the initial field at position $x=0$ and time $t$ as $\mathcal{E}(0, t)=$ $\frac{1}{2 \pi} \int_{\infty}^{\infty} \mathcal{F}[\mathcal{E}(0, t)](\omega) \exp (i \omega t) d \omega$. From this we get the field at $x$ as $\mathcal{E}(x, t)=\frac{1}{2 \pi} \int_{-\infty}^{\infty} \mathcal{F}[\mathcal{E}(x, t)](\omega) \exp (i \omega t) d \omega$. In addition, we assume that the medium is lossless, i.e., that $n(\omega)$ is real for all frequencies of the comb.

The Fourier transform of the field with respect to time is

$$
\begin{aligned}
\mathcal{F}[\mathcal{E}(x, t)](\omega) & \\
= & \pi \sum_{m=0}^{\infty} a_{m} \exp \left[-i\left(m \omega_{r}+\omega_{0}\right) n\left(m \omega_{r}+\omega_{0}\right) \frac{x}{c}\right] \\
& \times \delta\left(\omega-m \omega_{r}-\omega_{0}\right) \\
& +\pi \sum_{m=0}^{\infty} a_{m}^{*} \exp \left[i\left(m \omega_{r}+\omega_{0}\right) n\left(m \omega_{r}+\omega_{0}\right) \frac{x}{c}\right] \\
& \times \delta\left(\omega+m \omega_{r}+\omega_{0}\right),
\end{aligned}
$$

where we have used $n(-\omega)=n(\omega)$.
Consider now the interference of fields that have propagated over the distances $x_{1}$ and $x_{2}$, respectively. The total field at a given time is

$$
\mathcal{E}_{\text {tot }}\left(x_{1}, x_{2}, t\right)=\mathcal{E}\left(x_{1}, t\right)+\mathcal{E}\left(x_{2}, t\right) .
$$

A detector is used with a broad detection window implying $-\frac{T_{d}}{2}<t<\frac{T_{d}}{2}$ with $T_{d} \approx 1 \mathrm{~ms} \cong 10^{6} T_{r}$. We define

$$
\chi_{T_{d}}(t)= \begin{cases}\frac{1}{T_{d}} & \text { if }-\frac{T_{d}}{2}<t<\frac{T_{d}}{2}, \\ 0 & \text { otherwise }\end{cases}
$$

with the Fourier transform given by $\mathcal{F}\left[\chi_{T_{d}}\right](\omega)=\operatorname{sinc}\left(\frac{1}{2} \omega T_{d}\right)$. The signal measured by the slow detector is

$$
\begin{aligned}
P\left(x_{1}, x_{2}\right)= & \frac{1}{T_{d}} \int_{-T_{d} / 2}^{T_{d} / 2}\left(\left|\mathcal{E}\left(x_{1}, t\right)\right|^{2}+\left|\mathcal{E}\left(x_{2}, t\right)\right|^{2}\right) d t \\
& +\frac{2}{T_{d}} \operatorname{Re} \int_{-T_{d} / 2}^{T_{d} / 2} \mathcal{E}\left(x_{1}, t\right) \mathcal{E}\left(x_{2}, t\right)^{*} d t .
\end{aligned}
$$

For $i, j=1,2$ we can write the second integral as

$$
\begin{aligned}
\Gamma\left(x_{i}, x_{j}\right)= & T_{d} \operatorname{Re} \int_{-\infty}^{\infty} \chi_{T_{d}}(t) \mathcal{E}\left(x_{i}, t\right) \chi_{T_{d}}(t) \mathcal{E}\left(x_{j}, t\right)^{*} d t \\
= & \frac{T_{d}}{2 \pi} \operatorname{Re} \int_{-\infty}^{\infty} \mathcal{F}\left[\chi_{T_{d}}(t) \mathcal{E}\left(x_{i}, t\right)\right](\omega) \\
& \times \mathcal{F}\left[\chi_{T_{d}}(t) \mathcal{E}\left(x_{j}, t\right)\right]^{*}(\omega) d \omega,
\end{aligned}
$$

where in the last step we used the Parseval's theorem. Now we write

$$
\begin{aligned}
\mathcal{F}\left[\chi_{T_{d}}(t) \mathcal{E}\left(x_{i}, t\right)\right](\omega) & \frac{1}{2} \sum_{m=0}^{\infty} a_{m} \exp \left[-i\left(m \omega_{r}+\omega_{0}\right) n\left(m \omega_{r}+\omega_{0}\right) \frac{x_{i}}{c}\right] \\
& \times \operatorname{sinc}\left[\left(\omega-m \omega_{r}-\omega_{0}\right) \frac{T_{d}}{2}\right] \\
& +\frac{1}{2} \sum_{m=0}^{\infty} a_{m}^{*} \exp \left[i\left(m \omega_{r}+\omega_{0}\right) n\left(m \omega_{r}+\omega_{0}\right) \frac{x_{i}}{c}\right] \\
& \times \operatorname{sinc}\left[\left(\omega+m \omega_{r}+\omega_{0}\right) \frac{T_{d}}{2}\right] .
\end{aligned}
$$

Since $T_{d} \gg T_{r}=2 \pi / \omega_{r}$, the product of two functions $\mathcal{F}\left[\chi_{T_{d}}\right]\left(\omega \pm m \omega_{r} \pm \omega_{0}\right)$ with different $m$ 's is negligible. Therefore, by using the above equation, Eq. (5) can be written as

$$
\begin{aligned}
& \Gamma\left(x_{i}, x_{j}\right) \\
& \approx \frac{T_{d}}{2 \pi} \sum_{m=0}^{\infty}\left|a_{m}\right|^{2} \cos \left[\left(m \omega_{r}+\omega_{0}\right) n\left(m \omega_{r}+\omega_{0}\right) \frac{\left(x_{i}-x_{j}\right)}{c}\right] \\
& \times \int_{-\infty}^{\infty}\left[\operatorname{sinc}\left(\omega \frac{T_{d}}{2}\right)\right]^{2} d \omega \\
&= \sum_{m=0}^{\infty}\left|a_{m}\right|^{2} \cos \left[\left(m \omega_{r}+\omega_{0}\right) n\left(m \omega_{r}+\omega_{0}\right) \frac{\left(x_{i}-x_{j}\right)}{c}\right] .
\end{aligned}
$$

Here we have used $\int_{-\infty}^{\infty}\left[\operatorname{sinc}\left(\omega \frac{T_{d}}{2}\right)\right]^{2} d \omega=2 \pi / T_{d}$. Since the measured intensity will not depend explicitly on $x_{1}$ and $x_{2}$ we 

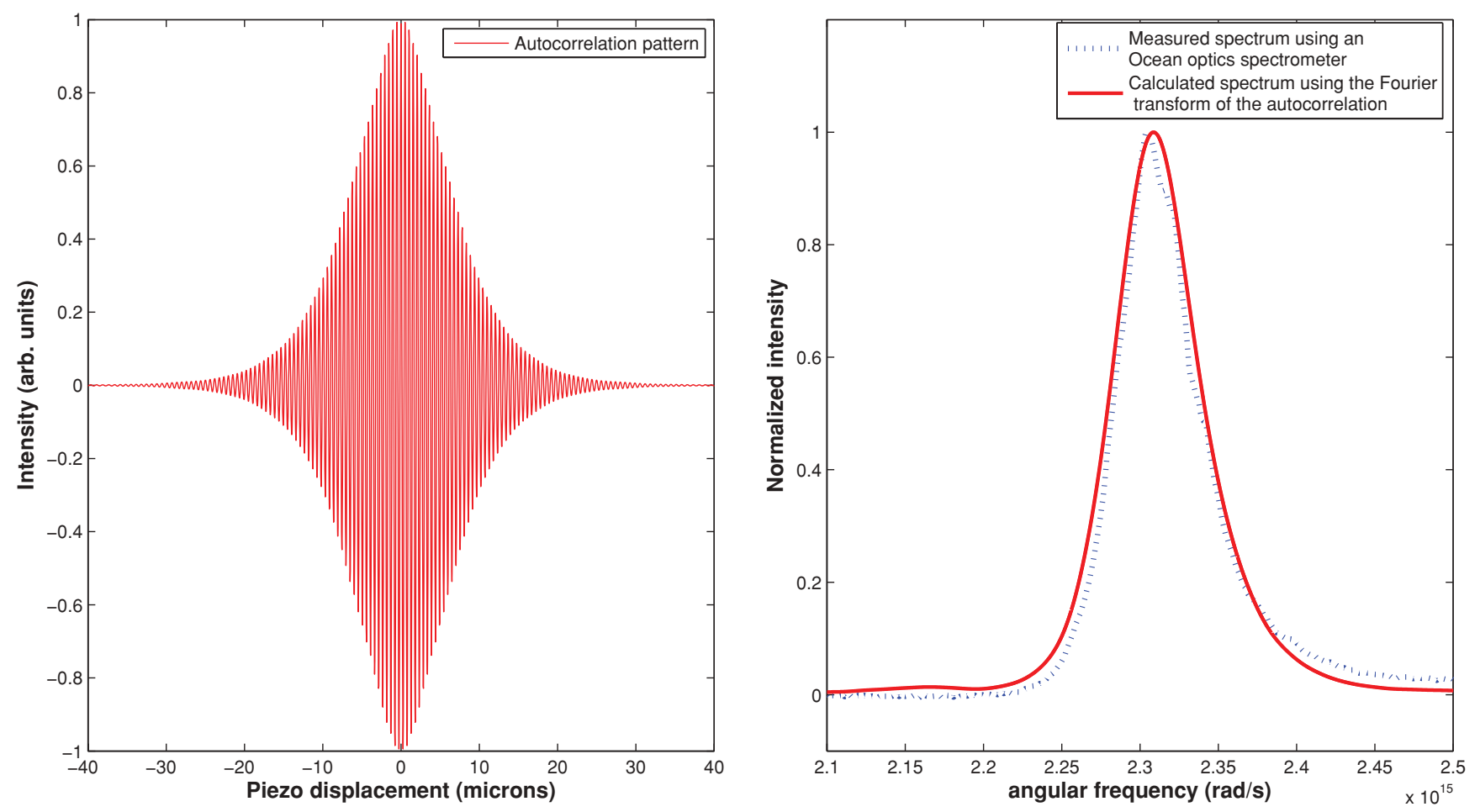

FIG. 2. (Color online) Left: Autocorrelation pattern. Right: Laser spectra directly measured, continuous line, compared with one retrieved from the autocorrelation, dotted line.

can take $X=x_{1}-x_{2}$ and write $\Gamma(X) \equiv \Gamma\left(x_{i}, x_{j}\right)$. Then the measured intensity is $P\left(x_{1}, x_{2}\right)=2 \Gamma(0)+2 \Gamma(X)$ where the cross-correlation, apart from the DC-background $2 \Gamma(0)$ and a factor of 2 , is given by

$$
\Gamma(X)=\sum_{m=0}^{\infty}\left|a_{m}\right|^{2} \cos \left[\left(m \omega_{r}+\omega_{0}\right) n\left(m \omega_{r}+\omega_{0}\right) \frac{X}{c}\right],
$$

here $\left|a_{m}\right|^{2}$ is defined as the power spectral density (PSD). This shows that the first-order cross-correlation function requires the knowledge of the source spectrum and not the pulse electric field. Numerical analysis based on Eq. (8) with comparison to measured data will be given in the next part of this section.

\section{B. Measured cross-correlations compared with numerical model}

In the present section we will compare numerically simulated cross-correlations using the results from the previous section with our measured data.

A general description of the experimental arrangement was given in the introduction of Sec II. The detailed measurement setup is described in Ref. [22]. The aim of this experiment was to demonstrate absolute distance metrology using a frequency-comb laser as a source in an unbalanced Michelson interferometer.

As mentioned earlier, analysis of the cross-correlation patterns to extract the distance requires an accurate knowledge of the position of the brightest fringe of the correlation pattern. The pulse propagating in the long arm of the interferometer acquires considerable chirp due to the dispersion in air leading to changes in shape and width of the resulting cross-correlation patterns. The asymmetry of the spectral profile of the pulses also plays a role in this. The spectrum of the laser source has been obtained from two separate measurements. A direct measurement was done using an Ocean Optics spectrometer. The spectrum was also retrieved from the measured first order autocorrelation pattern. We find good agreement between both the measured spectra, as shown in Fig. 2.

The spectral content of the initial pulse is the main input for the numerical model. Using the corrected updated Edlén's equation [29] for the refractive index of air, $8 \times 10^{4}$ spectral lines fitted to the profile of the spectrum of the laser, are propagated. Specific details of the laser, the spectra, and the pulse were mentioned in the previous section. The frequencycomb laser emits broadband pulses which, after traveling through different paths in the interferometer, superpose and form interference fringes. For path length differences which are an integer multiple of the laser cavity length, crosscorrelation patterns are calculated. For distance measurements, the brightest fringe position is used in data analysis, since a maximum fringe intensity indicates the occurrence of a maximum temporal coherence between superposed pulses. In vacuum, a maximum coherence indicates that the path length difference in the Michelson interferometer is exactly $q l_{p p}$ where $l_{p p}=2 \pi c / \omega_{r}$ and $q$ is an integer. Carrying out distance measurements in air requires a detailed knowledge of the dispersion relation. The phase delay is $v_{p}(\omega)=\omega / k(\omega)=$ $c / n(\omega)$, where $k(\omega)$ is the wave number and $n(\omega)$ is the refractive index given by the Edlén's equation. After a certain propagation length, the delay of the pulse envelope, the group delay, is equal to $v_{g}=d \omega / d k(\omega)=c / n_{g}$. Here $n_{g}$ is the group refractive index defined at the carrier frequency of the 


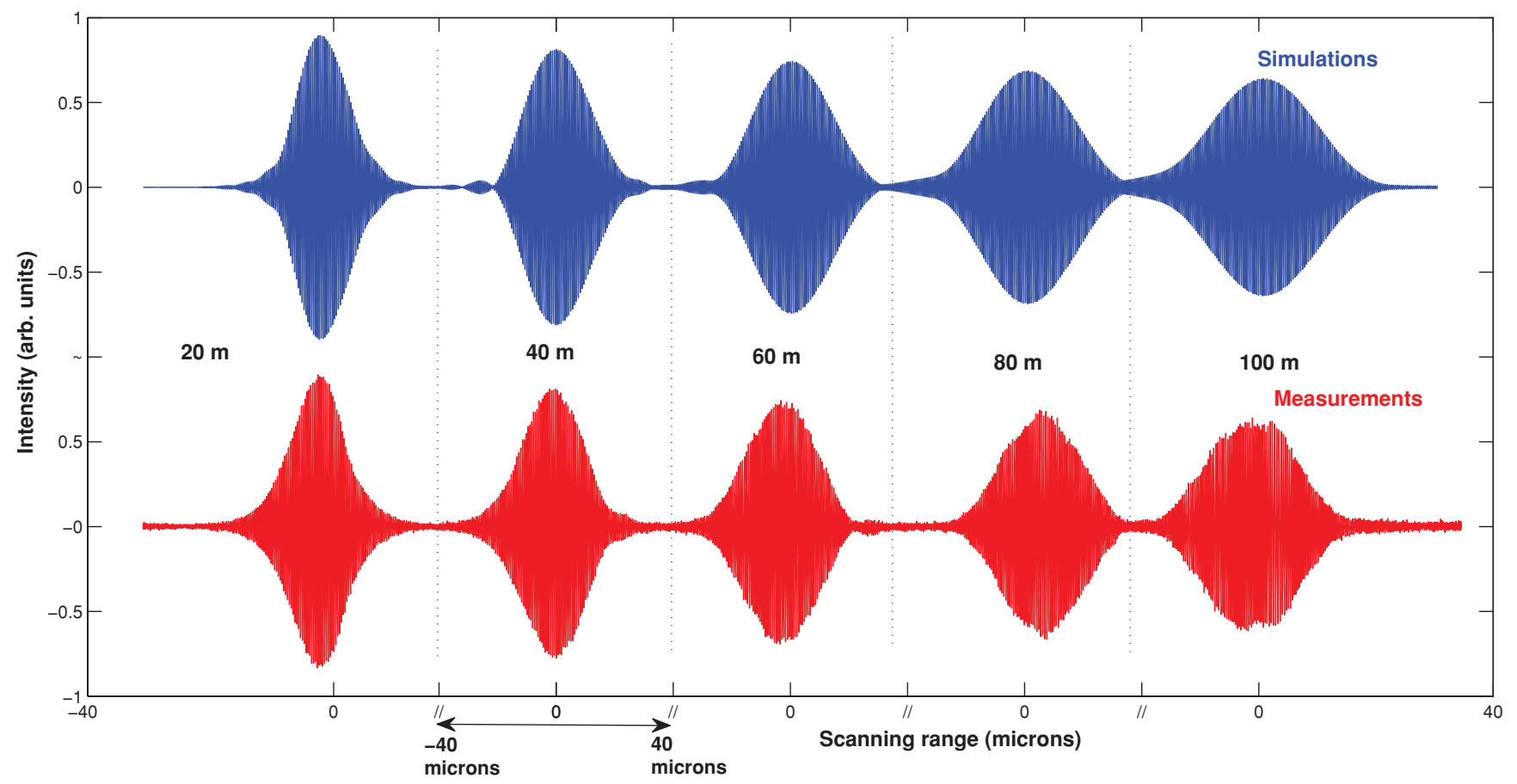

FIG. 3. (Color online) Measured patterns compared with simulation of various cross-correlation in air under the same environmental conditions.

pulse $\left(\omega_{c}\right)$, conventionally accepted as the frequency with the maximum intensity in the spectrum, and given by

$$
n_{g}=n\left(\omega_{c}\right)+\omega_{c}\left\{\frac{d[n(\omega)]}{d \omega}\right\}_{\omega_{c}} .
$$

For interferometric distance metrology, the maximum fringe visibility is reached at a given delay $L$. In vacuum $L=q l_{p p}$ and in a dispersive medium $L_{n}=q l_{p p} / n_{g}$. We would like to emphasize here that the position of $L_{n}$ does not coincide with the position of the maximum of the cross-correlation pattern. The formation of a cross-correlation after the pulse propagates in dispersive media does not depend only on the individual pulse path in one arm, but rather on the interference between pulses which have encountered different delays. The two positions coincide only when the medium is linearly dispersive.

Simulation using Eq. (8) and corresponding measured cross-correlation patterns are shown in Fig. 3. The simulated and measured cross-correlation patterns correspond to path length differences of $20,40,60,80$, and $100 \mathrm{~m}$ in air. In general, the shapes show good agreement. Simulated and measured patterns both show similar chirp and broadening. It can be seen that the numerical model can account for the effect of nonlinear dispersion on the pulses which have an asymmetric frequency spectrum. Full width at $1 / e$ of the maximum $\left(F \mathcal{W}_{1 / e}\right)$ of measured and simulated correlation patterns are given in Fig. 4. The comparison is done for 0 up to $200 \mathrm{~m}$ propagation in air. For short distances (less than $80 \mathrm{~m}), F \mathcal{W}_{1 / e}$ of both simulated and measured pattern agree very well. After $80 \mathrm{~m}$ of path length difference, a disagreement is observed. This difference is mostly due to the unpredictable effects of vibrations in the interferometer and air turbulence in the measurement room. A comparison between the numerical and experimental correlations is shown in Fig. 5 for the delay distances of 160 and $200 \mathrm{~m}$. From Figs. $5 \mathrm{c}$ and $5 \mathrm{f}$ we can clearly see that the measured patterns suffer from extra broadening compared to the simulated ones. We also observe that the chirp has stabilized and the correlations show only a linear broadening. To demonstrate this effect, let us consider $R_{H W}$ and $L_{H W}$ as the right and the left half width at $1 / e$ of the maximum, respectively (see Fig. 5a). We define the chirp ratio as follows:

$$
(\text { chirp ratio })=\frac{R_{H W}}{L_{H W}} .
$$

We plot this chirp ratio from the measured and simulated patterns in Fig. 6 for propagation from 0 to $200 \mathrm{~m}$ in air. From our simulations we see that the chirp reaches its maximum at a distance of $\sim 30 \mathrm{~m}$. After $30 \mathrm{~m}$, this ratio decays and tends asymptotically to a constant value for delays larger than $150 \mathrm{~m}$. We investigate these numerical and experimental observations rigorously in the next section.

\section{Numerical study of the correlation patterns as a function of power spectral density}

From Eq. (8) we can see that the formation of the crosscorrelation function depends on the spectral distribution of the laser source. In this section we discuss numerically the behavior of cross-correlation patterns using various types of power spectral densities (PSD's). The numerical study will be based on spectral distributions that are symmetric, e.g., Gaussian compared to distributions which are asymmetric and closer to measured spectra. 


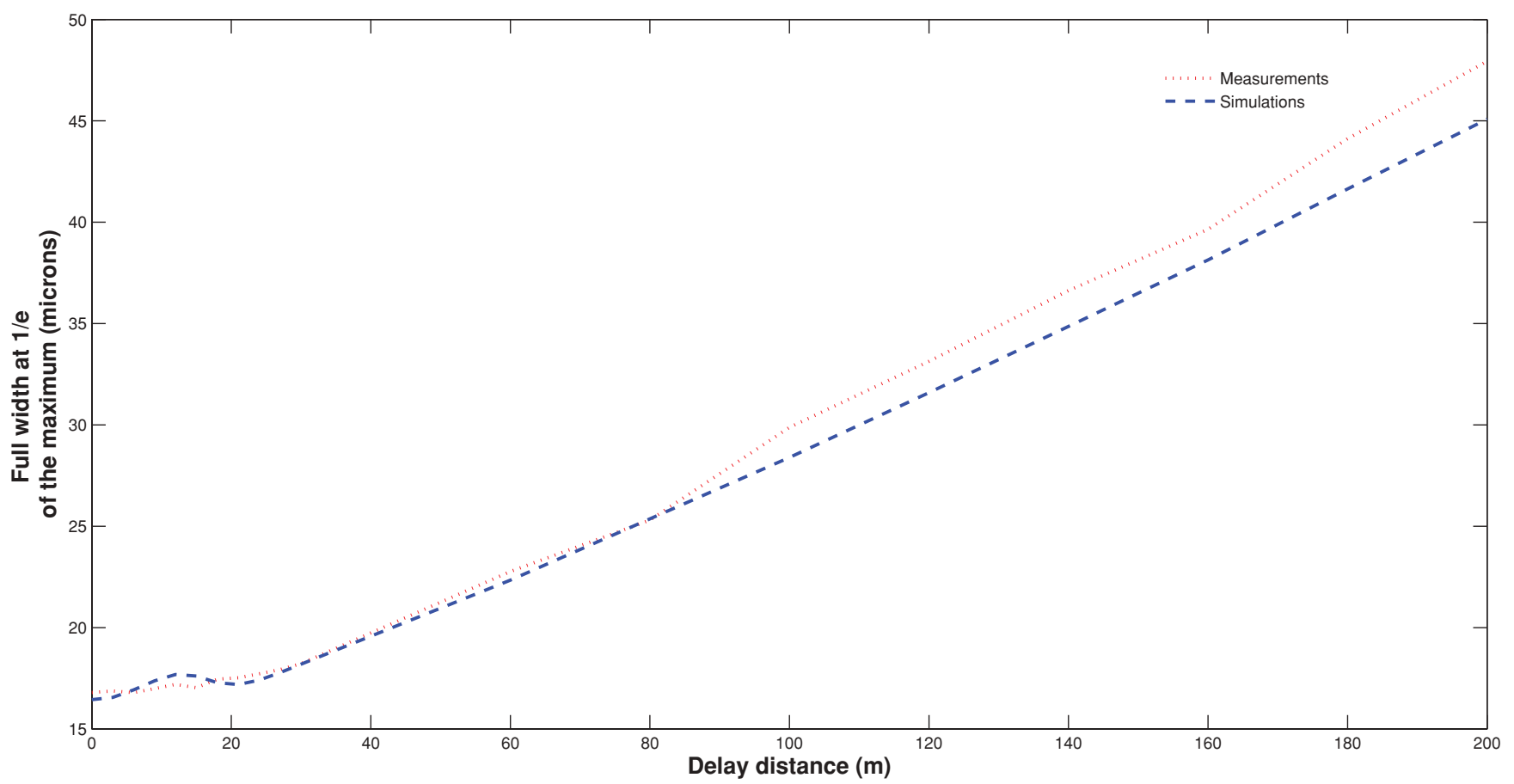

FIG. 4. (Color online) Comparison of the broadening of cross-correlation patterns between simulated, dashed line and measured, dotted line data.

In Fig. 7 we illustrate the cross-correlation patterns generated by various types of PSD's in two different media. An experimental asymmetric PSD is shown in Fig. 7a along with a $\operatorname{sech}^{2}$ and a Gaussian PSD. Using these PSD's we have simulated the cross-correlation function in air and in BK7 glass by taking two distances, one relatively short and the other relatively long.
In the cases where the PSD's are symmetric, the cross-correlations remain symmetric without showing any shift with respect to the position of the brightest fringe. Only when the PSD is asymmetric as in a general experiment, the position of the brightest fringe is not in the center of the crosscorrelation. In addition, the shape of the cross-correlations is distorted.
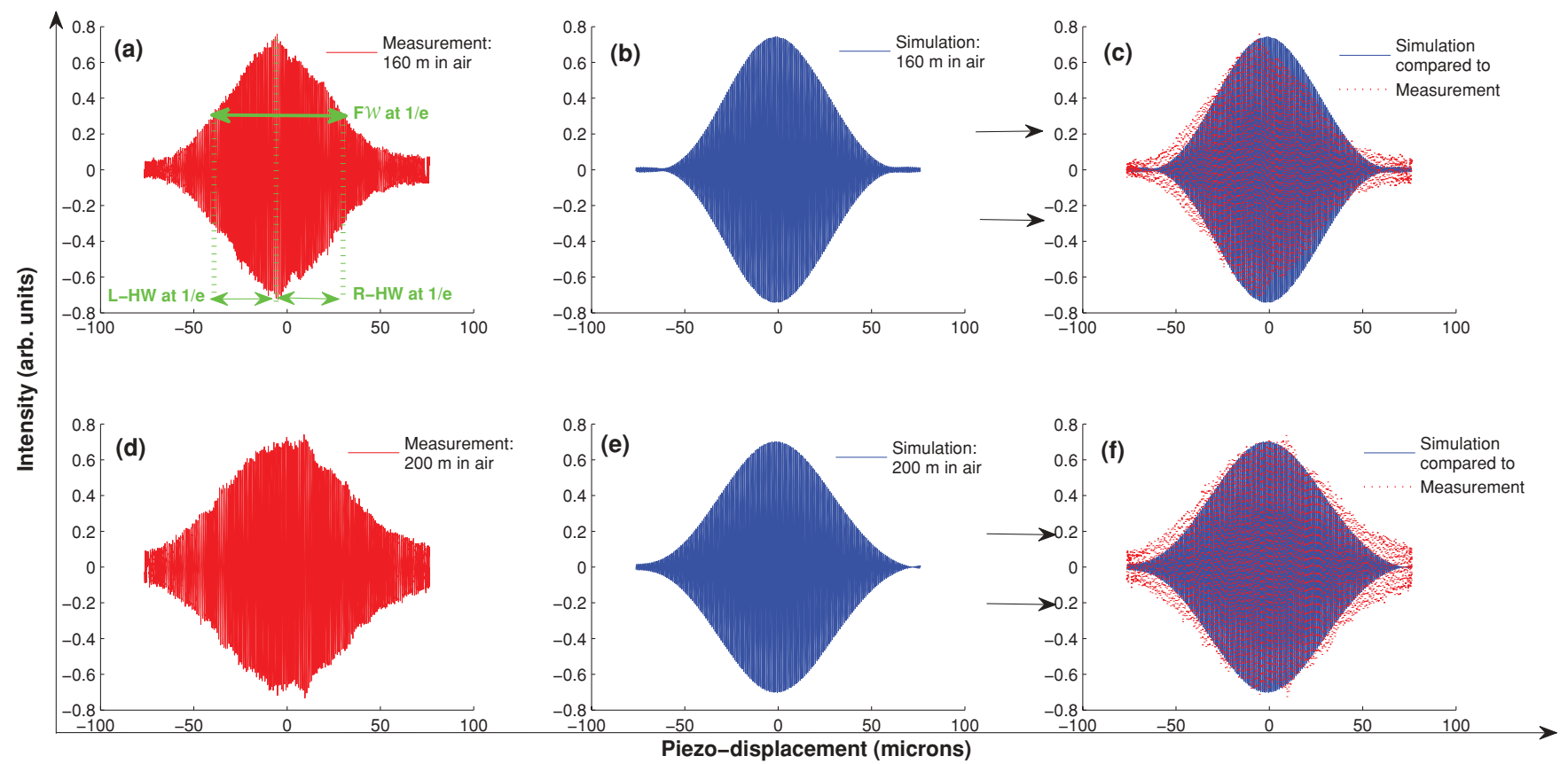

FIG. 5. (Color online) Comparison between measured and simulated cross-correlation patterns for $160 \mathrm{~m}$ and $200 \mathrm{~m}$ in air to evaluate shape distortion arising from asymmetric spectra. 


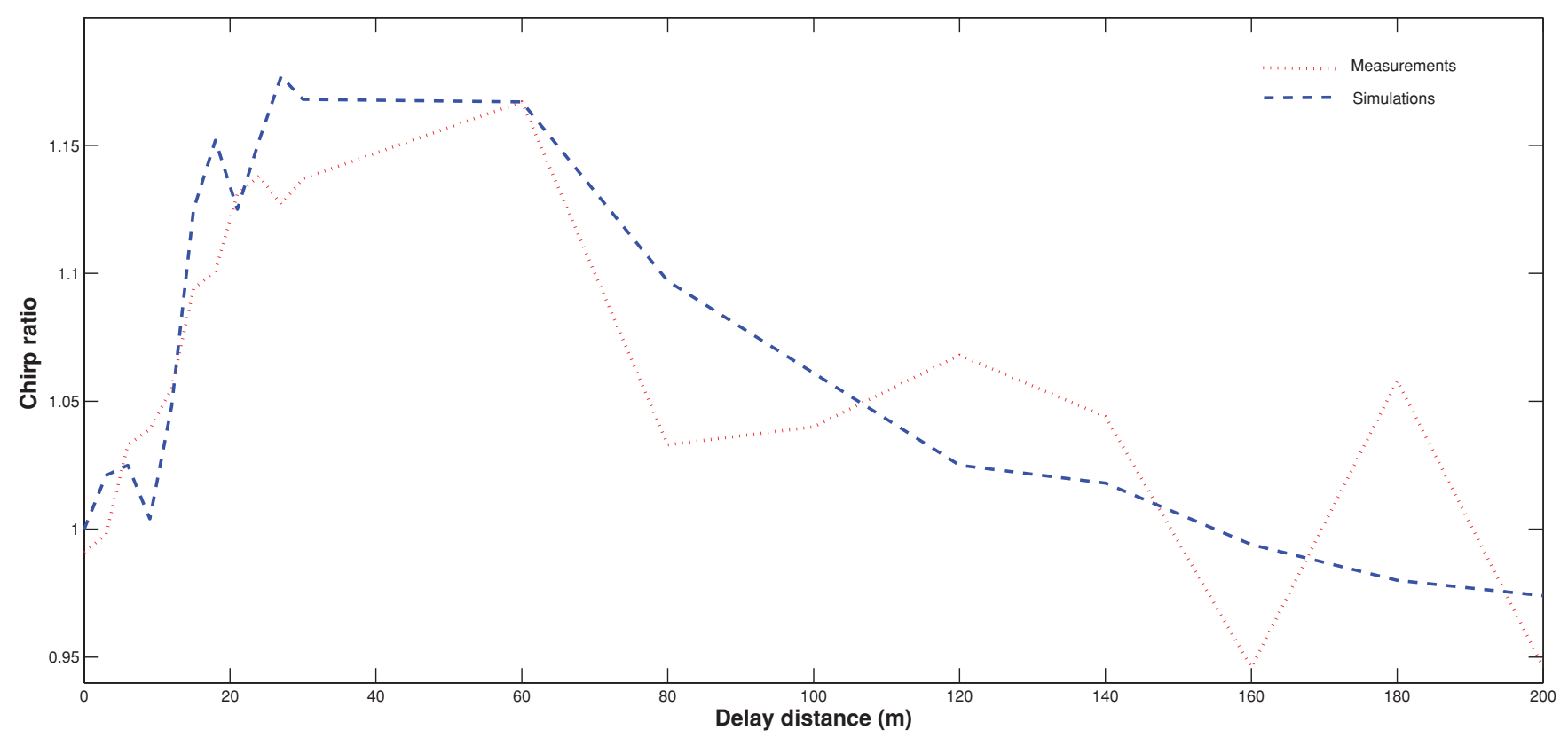

FIG. 6. (Color online) Analysis of shape distortion of measured, dotted line and simulated, dashed line, cross-correlation patterns.

To illustrate the effects of asymmetric PSD's in nonlinear dispersive media, we will take the example of an asymmetric Gaussian pulse defined by

$$
a(\omega)= \begin{cases}\exp \left[-\frac{\left(\omega-\omega_{c}\right)^{2}}{2 \sigma^{2}\left(1-a_{r}\right)^{2}}\right] & \omega \leqslant \omega_{c} \\ \exp \left[-\frac{\left(\omega-\omega_{c}\right)^{2}}{2 \sigma^{2}\left(1-a_{l}\right)^{2}}\right] & \omega>\omega_{c}\end{cases}
$$

where $a_{r}$ and $a_{l}$ are the decay parameters for the regions to the right and left of $\omega_{c}$, respectively. Here, $\omega_{c}$ is the frequency of the PSD where intensity is maximum. We consider two cases, one, $a_{l}=0$ and $a_{r}=0.5$ and the second $a_{r}=0$ and $a_{l}=0.5$. The corresponding PSD's and the cross-correlation patterns simulated for two different distances are shown in Fig. 8. It is clearly seen that the behavior of the first-order cross-correlation pattern is strongly affected by the shape of

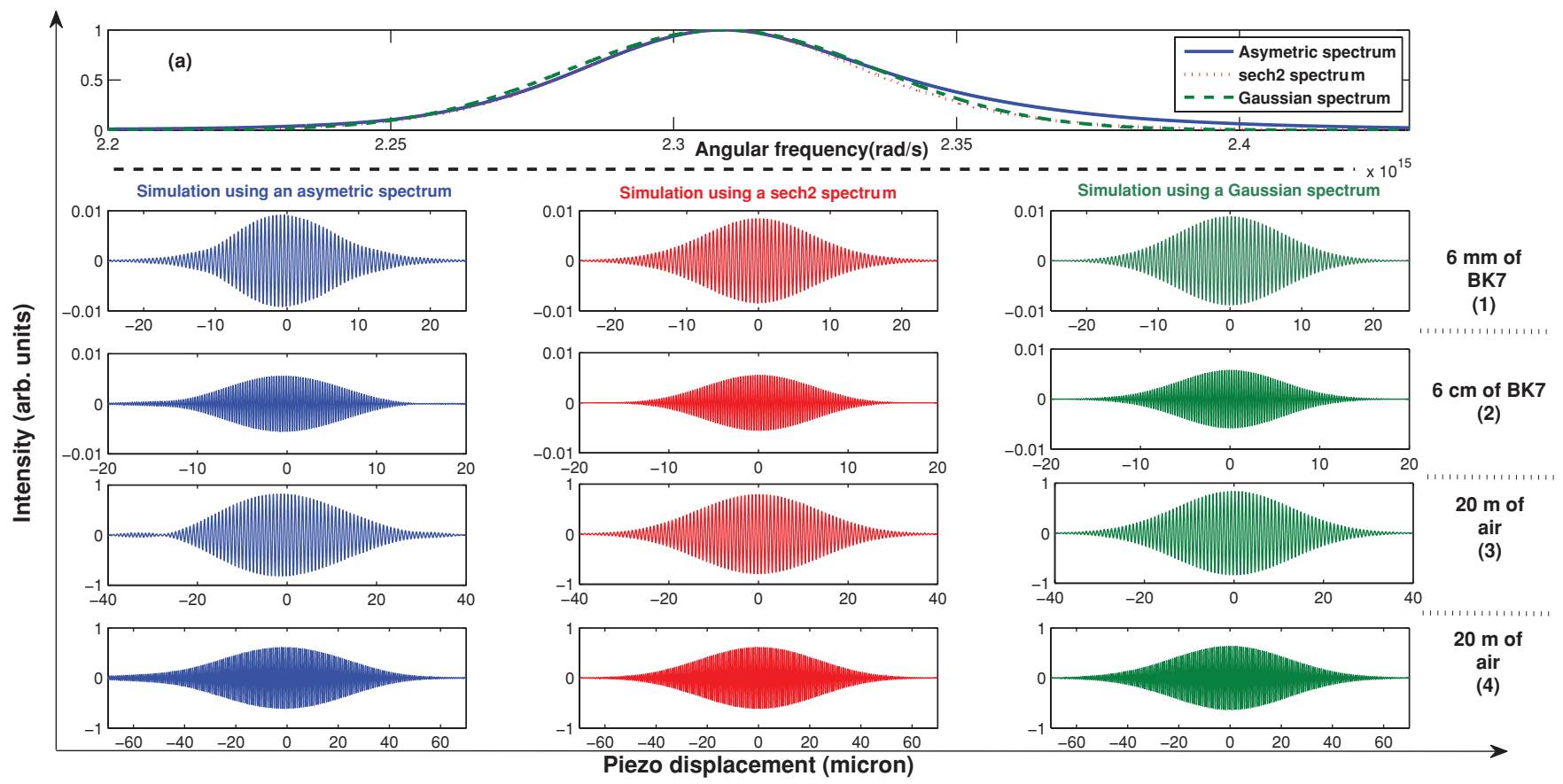

FIG. 7. (Color online) Comparison between cross-correlations in different dispersive media generated using symmetric, Gaussian (dashed line), $\operatorname{sech}^{2}$ (dotted line) and asymmetric, continuous line, power spectral densities. 


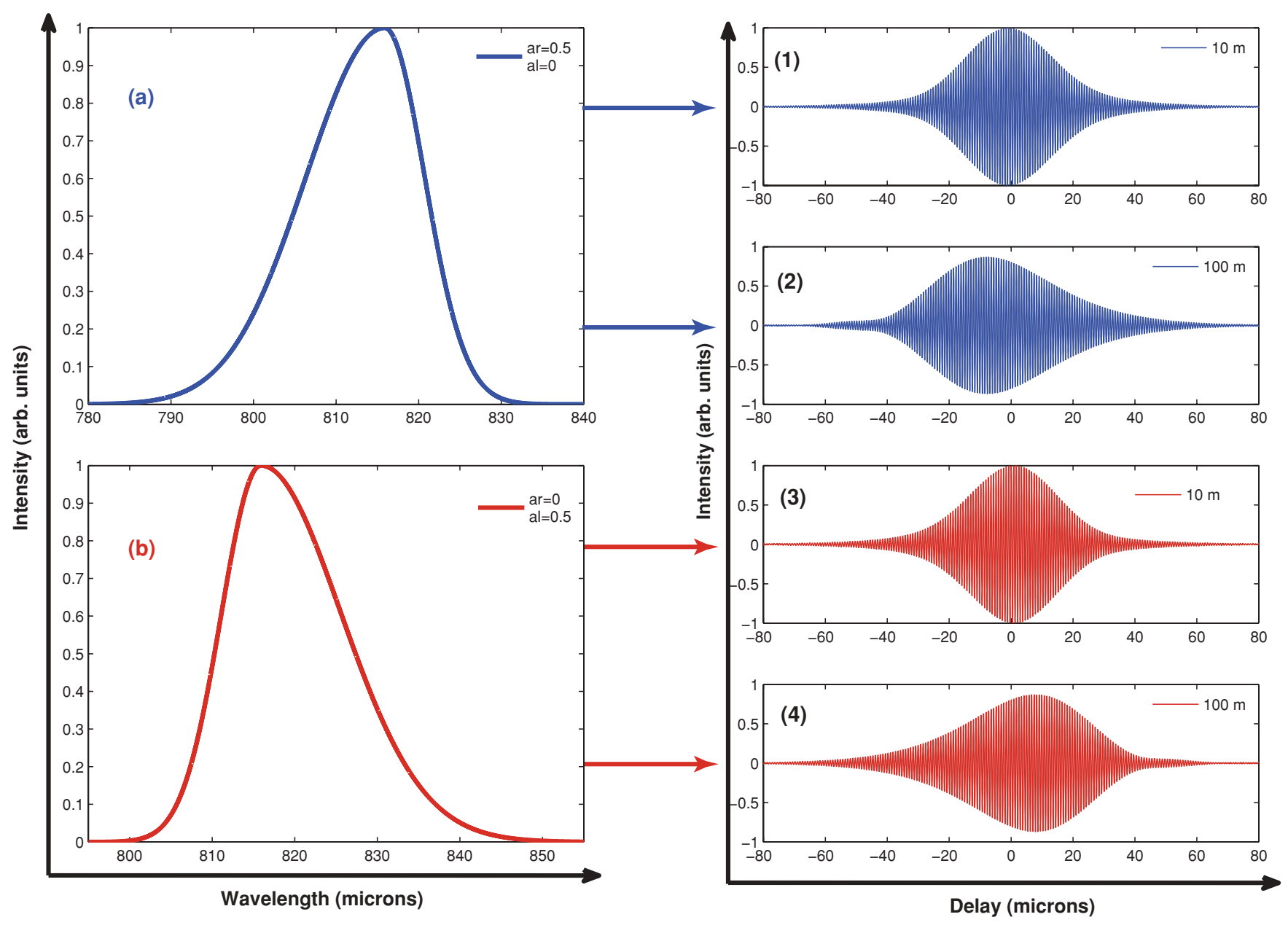

FIG. 8. (Color online) (a) Right asymmetric Gaussian PSD from Eq. (11) used to compute cross-correlations for distances of $10 \mathrm{~m}$ and $100 \mathrm{~m}$. (b) Left asymmetric Gaussian PSD from Eq. (11) used to compute cross-correlations for distances of $10 \mathrm{~m}$ and $100 \mathrm{~m}$.

the PSD. It is important to observe that the position of the absolute maximum of the cross-correlation is shifted.

Finally, we would like to mention the effect of the environmental parameters, i.e., temperature, pressure, and humidity of air. Environmental fluctuation has the biggest influence on the accuracy of the measured distance. In Fig. 9, we consider the example of simulated correlation patterns at $60 \mathrm{~m}$ pulse propagation for subtle changes in the three relevant parameters. The position of the brightest fringe of the correlation pattern obtained for $20^{\circ} \mathrm{C}, 1013.25 \mathrm{hPa}$, and $45 \%$ humidity is taken as a reference. We observe that a $0.2^{\circ} \mathrm{C}$ temperature variation shifts the brightest fringe of the correlation pattern by $5.36 \mu \mathrm{m}$. A pressure increase of $1 \mathrm{hPa}$ results in a $10.34 \mu \mathrm{m}$ shift. Humidity variation of $1 \%$ shows a shift of $0.527 \mu \mathrm{m}$. These results are shown in Fig. 9. The simulations show that the correlations patterns only shift without any extra linear broadening or chirp.

\section{CONTINUOUS MODEL FOR CROSS-CORRELATION FUNCTIONS}

In this section we study fringe positioning and asymptotic convergence of correlation patterns for large delay distances.
The cross-correlation is a sum of all harmonics of a slowly varying function, the PSD, multiplied by an oscillating phase factor. We would now like to extend the discrete model to a continuous one by using an integral in the formulation of the cross-correlation function $\Gamma(X)$, instead of a series as in Eq. (8). Clearly the PSD, $\left|a_{m}\right|^{2}$, in Eq. (8) varies slowly with $m$; however the cosine function does not, and therefore it is not valid to directly replace the sum by an integral. Instead, the corresponding integral must be found via other methods. In this section we use the Poisson summation formula to write a series of all possible cross-correlation functions for the chosen PSD and dispersive media. From this series we obtain the integral formulation of the cross-correlation function for a specific delay distance.

We would like to note here that the transition from a discrete to a continuous model can be expressed using simpler methods. This is only true if the mode-locked laser is operating at a repetition frequency lower than few tens of megahertz where the contribution of the offset frequency is insignificant. Recent developments in femtosecond laser technology allow for faster repetition frequencies with fewer modes [30]. There are also examples of experiments where the frequency comb has been filtered with a Fabry-Perot cavity [20]. In addition, 


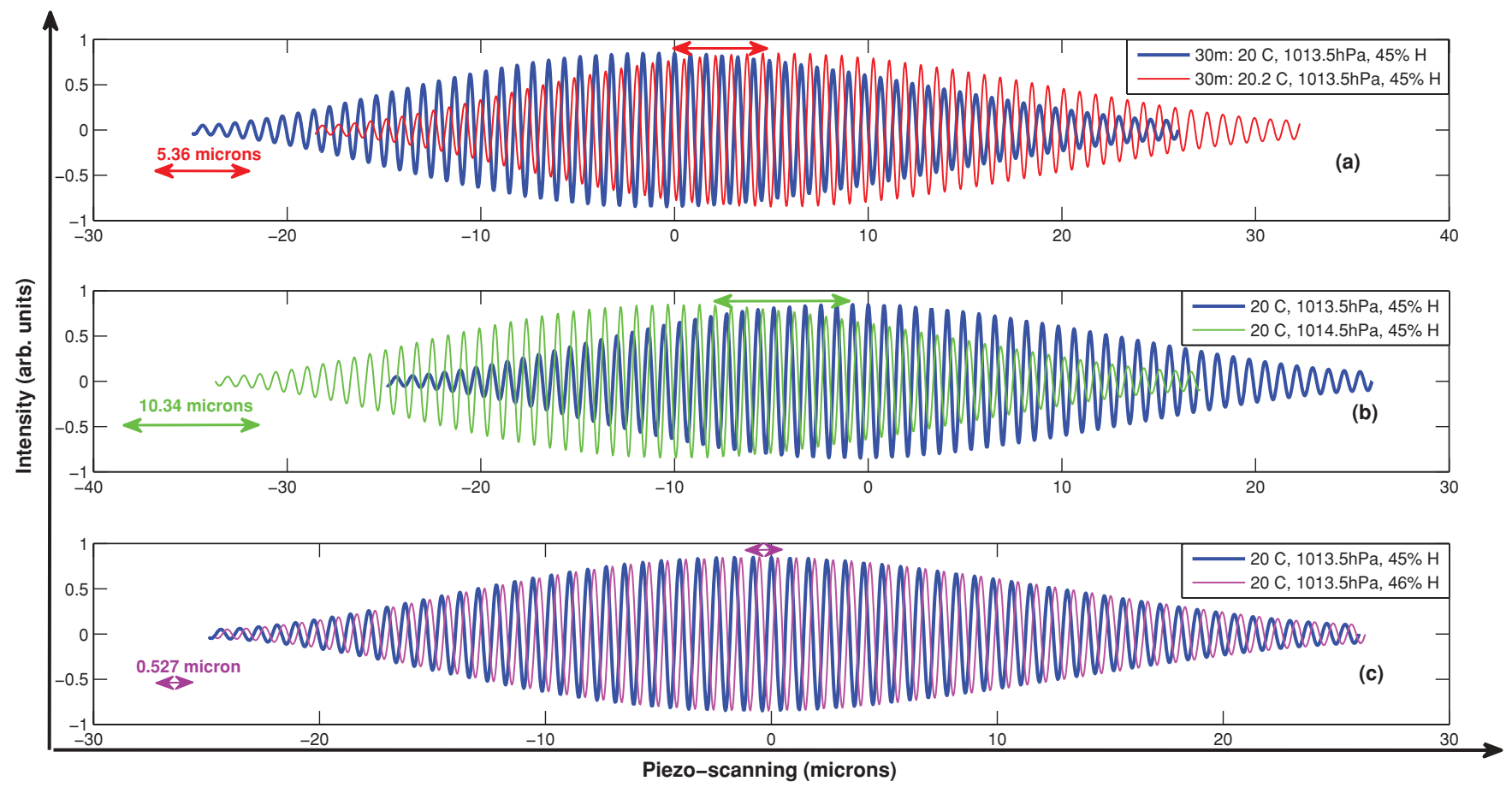

FIG. 9. (Color online) The effect of variation in the environmental parameters (a) temperature, (b) pressure, and (c) humidity on the cross-correlation pattern. Simulation was done for a distance of $30 \mathrm{~m}$. Reference conditions are indicated by the thick line, $20^{\circ} \mathrm{C}, 1013.5 \mathrm{hPa}$, $45 \%$ humidity, variations are indicated by the thin line.

in the limit of large delay distances the precise values of the offset and repetition frequencies become important since particular frequencies contribute to the formation of specific fringes in the cross-correlation. In these cases it would be invalid to neglect the offset frequency. Therefore, we have developed a general rigorous formula of the cross-correlation function applied to any type of train of pulses emitted from a frequency-comb laser. This was made possible by using the Poisson summation formula.

Let us extend the definition of the discrete coefficients $a_{m}$ from the definition of the electric field in Sec. II A to continuous variables, where $\mathcal{E}^{p}(0, t)=\sum_{m=0}^{\infty} a_{m} \exp \left(i m \omega_{r} t\right)$. To be able to map a continuous spectrum to the discrete one we need to provide this electric field with a finite support $\left[-T_{r} / 2, T_{r} / 2\right]$ as $\mathcal{E}_{T_{r}}^{p}(0, t)=\mathcal{E}^{p}(0, t) \chi_{\left[-\frac{T_{r}}{2}, \frac{T_{r}}{2}\right]}(t)$. Here the $\chi$ function is defined similarly to Eq. (3) with a time interval equal to $T_{r}$ which is the interpulse time interval. The Fourier transform of $\mathcal{E}_{T_{r}}^{p}(0, t)$ is given by $\mathcal{F}\left[\mathcal{E}_{T_{r}}^{p}(0, t)\right](\omega)=\sum_{m=0}^{\infty} a_{m} \operatorname{sinc}[(\omega-$ $\left.\left.m \omega_{r}\right) \frac{T_{r}}{2}\right]$. In particular, $\mathcal{F}\left[\mathcal{E}_{T_{r}}^{p}(0, t)\right]\left(m \omega_{r}\right)=a_{m}$. We define for real $\omega$, i.e., $-\infty<\omega<\infty$, the power spectral density as

$$
S\left(\omega+\omega_{0}\right)=\left|\mathcal{F}\left[\mathcal{E}_{T_{r}}^{p}(0, t)\right](\omega)\right|^{2}
$$

This implies $S\left(m \omega_{r}+\omega_{0}\right)=\left|a_{m}\right|^{2}$. By substituting $S\left(m \omega_{r}+\right.$ $\omega_{0}$ ) into Eq. (8) we have

$$
\Gamma(X)=\sum_{m=0}^{\infty} S\left(m \omega_{r}+\omega_{0}\right) \cos \left[\left(m \omega_{r}+\omega_{0}\right) n\left(m \omega_{r}+\omega_{0}\right) \frac{X}{c}\right] .
$$

Using $\bar{n}$ as the mean refractive index, we obtain

$$
\begin{aligned}
\Gamma(X)= & \frac{1}{2} \sum_{m=0}^{\infty} S\left(m \omega_{r}+\omega_{0}\right) \\
& \times \exp \left\{i\left(m \omega_{r}+\omega_{0}\right)\left[n\left(m \omega_{r}+\omega_{0}\right)-\bar{n}\right] \frac{X}{c}\right\} \\
& \times \exp \left[i \omega_{0} \frac{X}{c} \bar{n}\right] \exp \left[i m \omega_{r} \frac{X}{c} \bar{n}\right] \\
& +\frac{1}{2} \sum_{m=0}^{\infty} S\left(m \omega_{r}+\omega_{0}\right) \\
& \times \exp \left\{-i\left(m \omega_{r}+\omega_{0}\right)\left[n\left(m \omega_{r}+\omega_{0}\right)-\bar{n}\right] \frac{X}{c}\right\} \\
& \times \exp \left[-i \omega_{0} \frac{X}{c} \bar{n}\right] \exp \left[-i m \omega_{r} \frac{X}{c} \bar{n}\right] .
\end{aligned}
$$

Now we define

$$
\mathcal{H}_{X}(\omega)=\left\{\begin{array}{l}
S\left(\omega+\omega_{0}\right) \exp \left\{i\left(\omega+\omega_{0}\right)\left[n\left(\omega+\omega_{0}\right)-\bar{n}\right] \frac{X}{c}\right\} \exp \left[i \omega_{0} \bar{n} \frac{X}{c}\right], \quad \omega \geqslant 0 \\
S\left(-\omega+\omega_{0}\right) \exp \left\{i\left(\omega-\omega_{0}\right)\left[n\left(-\omega+\omega_{0}\right)-\bar{n}\right] \frac{X}{c}\right\} \exp \left[-i \omega_{0} \bar{n} \frac{X}{c}\right], \quad \omega \leqslant 0
\end{array}\right.
$$


and

$$
\mathcal{H}_{X}(0)=S\left(\omega_{0}\right) \cos \left[\omega_{0} n\left(\omega_{0}\right) \frac{X}{c}\right]
$$

Then Eq. (14) becomes

$$
\begin{aligned}
\Gamma(X)= & \mathcal{H}_{X}(0)+\frac{1}{2} \sum_{m=1}^{\infty} \mathcal{H}_{X}\left(m \omega_{r}\right) \exp \left[i m \omega_{r} \frac{X}{c} \bar{n}\right] \\
& +\frac{1}{2} \sum_{m=1}^{\infty} \mathcal{H}_{X}\left(-m \omega_{r}\right) \exp \left[-i m \omega_{r} \frac{X}{c} \bar{n}\right] .
\end{aligned}
$$

The intensity $S\left(\omega_{0}\right)$ is negligibly small, so we may replace $\mathcal{H}_{X}(0)$ by $\frac{1}{2} \mathcal{H}_{X}(0)$. Thus, with a negligibly small error we can write

$$
\Gamma(X)=\frac{1}{2} \sum_{m=-\infty}^{\infty} \mathcal{H}_{X}\left(m \omega_{r}\right) \exp \left[i m \omega_{r} \frac{X}{c} \bar{n}\right] .
$$

This form of the correlation is convenient for the application of the Poisson summation formula. Thus with $h_{X}(t)=$ $\frac{1}{2 \pi} \int_{-\infty}^{\infty} \mathcal{H}_{X}(\omega) \exp (i \omega t) d \omega$ the inverse Fourier transform of $\mathcal{H}_{X}$, it holds that

$$
\begin{aligned}
\Gamma(X) & =\frac{1}{2} \sum_{m=-\infty}^{\infty} \mathcal{F}\left[h_{X}(t)\right]\left(m \omega_{r}\right) \exp \left[i m \omega_{r} \frac{X}{c} \bar{n}\right] \\
& =\frac{1}{2} \sum_{m=-\infty}^{\infty} \mathcal{F}\left[h_{X}\left(t+\frac{X}{c} \bar{n}\right)\right]\left(m \omega_{r}\right) \\
& =\frac{1}{2} T_{r} \sum_{\ell=-\infty}^{\infty} h_{X}\left(\ell T_{r}+\frac{X}{c} \bar{n}\right) .
\end{aligned}
$$

Here the Poisson summation formula $\sum_{m=-\infty}^{\infty} \mathcal{F}\left[h_{X}\right]$ $\left(m \omega_{r}\right)=\frac{2 \pi}{\omega_{r}} \sum_{\ell=-\infty}^{\infty} h_{X}\left(\frac{2 \pi}{\omega_{r}} \ell\right)$ with $\omega_{r}=\frac{2 \pi}{T_{r}}$ has been used. This series expression for $\Gamma(X)$ in Eq. (19) reduces to at most a single term when $h_{X}$ has support length $\leqslant T_{r}$.

The pulse propagating in the long arm undergoes substantial broadening in the dispersive media. This in turn broadens the cross-correlation function. In the above analysis we are in the regime where the extent of the cross-correlation function is still smaller than the laser cavity length, in other words the intensity observed by the detector at the upper and lower bounds of the finite support, [-T $\left.-T_{r} / 2, T_{r} / 2\right]$, is negligible. We can demonstrate that the series in Eq. (19) contains at most one dominant term $(\ell)$. From a physical point of view, the integer $\ell$ denotes the multiple of the laser cavity length at a given delay distance $X$ and $\ell T_{r}$ is the propagation time of a pulse in vacuum. In the case where $X>0$ the integer $\ell$ must be negative. We would like to define the time variable, $t$, as $t \equiv \bar{n} \frac{X}{c}\left(\bmod T_{r}\right)$. Using this, for a given $\ell$, Eq. (19) can be written as

$$
\Gamma(X)=\frac{T_{r}}{2} h_{X}(t) .
$$

Expressing $h_{X}(t)$ in terms of the PSD and changing - $\omega$ into $\omega \in[0, \infty]$, we get

$$
\begin{aligned}
h_{X}(t)= & \frac{1}{\pi} \int_{0}^{\infty} S\left(\omega+\omega_{0}\right) \cos \left\{\left(\omega+\omega_{0}\right)\left[n\left(\omega+\omega_{0}\right)-\bar{n}\right] \frac{X}{c}\right. \\
& \left.+\omega_{0} \bar{n} \frac{X}{c}+\omega t\right\} d \omega .
\end{aligned}
$$

Equation (21) states that for any arbitrary delay distance $X$ a cross-correlation pattern $h_{X}(t)$ can be obtained by varying the time delay, $t$, where $-T_{r} / 2 \leqslant t \leqslant T_{r} / 2$. In practice, this time, $t$, can be obtained by setting up a scanning short arm which has a total length of the laser cavity.

\section{POSITION OF THE BRIGHTEST FRINGE AFTER PROPAGATION IN NONLINEAR DISPERSIVE MEDIA}

The shift of the brightest fringe due to dispersion will be discussed where the refractive index of the medium is limited to its quadratic approximation. This quadratic approximation has the mathematical advantage that the Fresnel transform can be used. Also it has been shown that optimal results can be obtained with the quadratic or the cubic dispersion approximation and including higher order terms does not improve the accuracy [26]. The following analysis will be based on the method introduced by Jones [28] for an electromagnetic pulse in dispersive media.

We aim to derive an expression for the cross-correlation using the properties of the autocorrelation. Our analysis starts from Eq. (21) where we set $X=0$. This is the case of an interferometer at equal arms, i.e., an autocorrelator. In this case, we obtain

$$
h_{X=0}(t)=\frac{1}{\pi} \int_{0}^{\infty} S\left(\omega+\omega_{0}\right) \cos (\omega t) d \omega .
$$

For convenience we separate the time variable of the autocorrelation and the cross-correlation. Let $\tau_{c}$ be the full duration of the initial autocorrelation, where $\tau_{c}<T_{r}$. The function $h_{X=0}\left(t_{0}\right)$ is real, even and vanishes outside an interval $\left[-\frac{1}{2} \tau_{c}, \frac{1}{2} \tau_{c}\right]$. The Wiener-Khintchin theorem states that the autocorrelation and the power spectral density are a Fourier transform pair, hence for $\omega>0$ we can write $S\left(\omega+\omega_{0}\right)=\int_{-\infty}^{\infty} h_{X=0}\left(t_{0}\right) \cos \left(\omega t_{0}\right) d t_{0}$. Using this in Eq. (21) and interchanging integrals, we obtain

$$
\begin{aligned}
h_{X}(t)= & \frac{1}{\pi} \int_{-\infty}^{\infty} h_{X=0}\left(t_{0}\right) d t_{0} \\
& \times \int_{0}^{\infty} \cos \left[k\left(\omega+\omega_{0}\right) X-\omega \bar{n} \frac{X}{c}+\omega t\right] \cos \left(\omega t_{0}\right) d \omega,
\end{aligned}
$$

where $k\left(\omega+\omega_{0}\right)=n\left(\omega+\omega_{0}\right)\left(\omega+\omega_{0}\right) / c$. We use the fact the the medium has a quadratic dispersion relation which can be expressed in terms of a Taylor expansion that is twice differentiable in the neighborhood of the maximum frequency of the spectrum $\omega_{c}$, also known as the carrier frequency. This allows us to write $k\left(\omega+\omega_{0}\right)=\alpha \omega^{2}+\beta \omega+\gamma$. We now consider the case of large delay distance $X$. To be precise, we assume that $X$ is so large that for all $t_{0}$ in the support $\left[-\tau_{c} / 2,-\tau_{c} / 2\right]$ of $h_{X=0}\left(t_{0}\right)$, we have $\frac{t_{0}}{2 \sqrt{\alpha X}} \ll 1$. Using this 


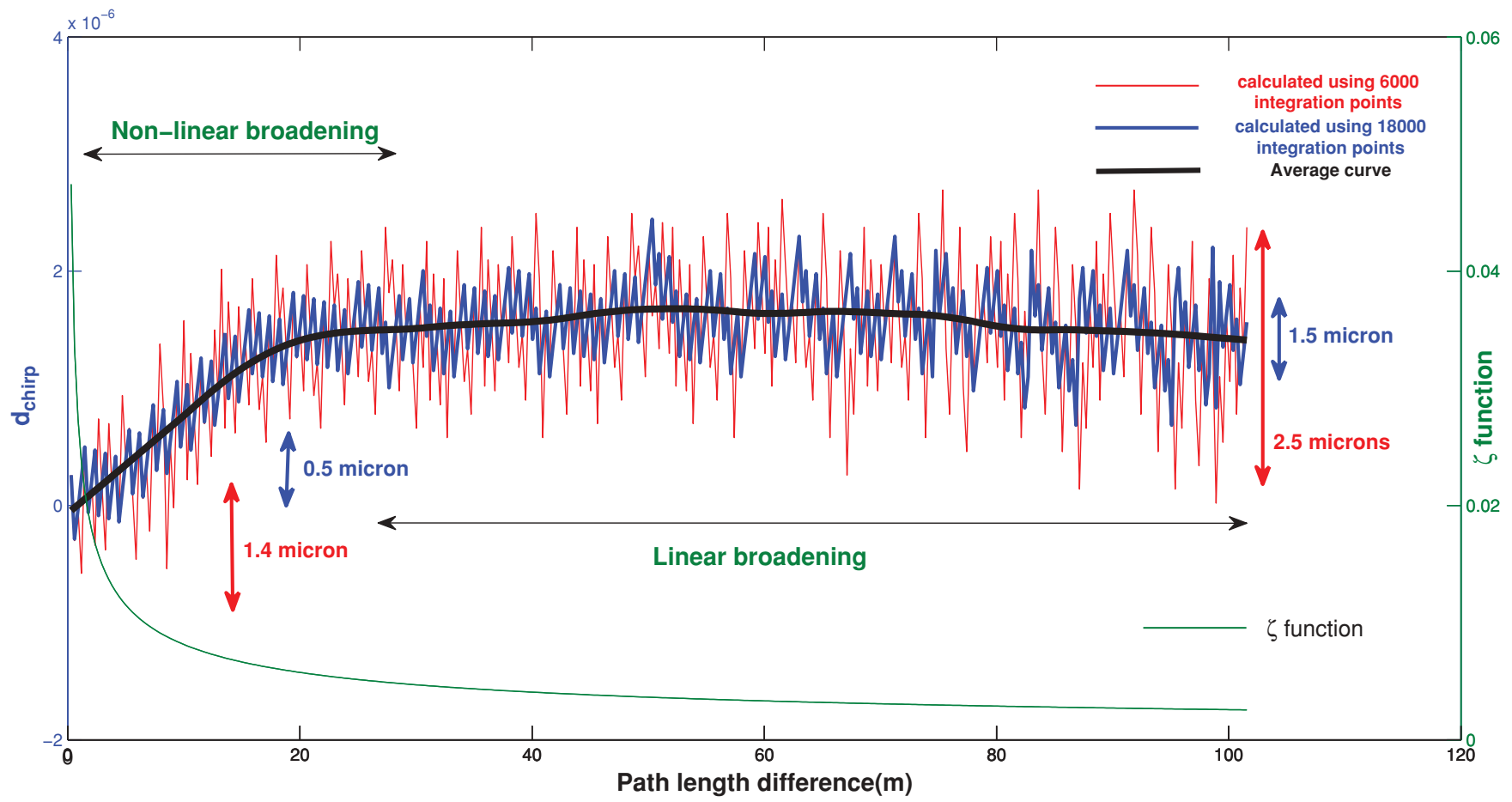

FIG. 10. (Color online) Shift of the position of the fringe having maximum visibility as a function of the path length difference. The $\zeta$ function is also plotted, showing the nonlinear dispersion depth.

we can derive an expression for $h_{X}(t)$ in a simple integral form as

$$
\begin{aligned}
h_{X}(t)= & \frac{R}{2 \pi} \sqrt{\frac{\pi}{2 \alpha X}} \cos \left[\gamma X-\frac{\left(\beta X-\bar{n} \frac{X}{c}+t\right)^{2}}{4 \alpha X}+\theta\right] \\
& \left.\times \int_{-\infty}^{\infty} d t_{0} h_{X=0}\left(t_{0}\right)\right] \cos \left[\frac{\beta X-\bar{n} \frac{X}{c}+t}{2 \alpha X} t_{0}\right] .
\end{aligned}
$$

The details of this calculation are given in the Appendix. In the above equation $R$ and $\theta$ are parameters dependent on the dispersion properties of the medium and are also explained in the Appendix. We observe that the time scale $\sqrt{4 \alpha X}$ plays a role in the distortion of the cross-correlation. Therefore, we can define a dimensionless function $\zeta(X)$ as

$$
\zeta(X)=\frac{\tau_{c}}{\sqrt{(4 \alpha X)}}=\frac{\tau_{c}}{\sqrt{\frac{2}{c}\left[2 \frac{d n(\omega)}{d \omega}+\omega \frac{d^{2} n(\omega)}{d \omega^{2}}\right]_{\omega=\omega_{0}} X}} .
$$

The constant $\tau_{c}$ can be simply obtained either from the Fourier transform of the PSD or from experimental measurements using an autocorrelator. The function $\zeta(X)$ is plotted for various delay distances in Fig. 10.

In order to study the variation of the position of the brightest fringe in dispersive media we have simulated a series of crosscorrelation patterns from 0 to $100 \mathrm{~m}$ delay in air with steps of one cavity length $(\sim 30 \mathrm{~cm})$. For each pattern, the position of the maximum fringe visibility is recorded, we refer to this position as $d_{m c}$. Now we consider the distance $d_{n_{g}}$ defined by $d_{n_{g}}=\frac{q l_{p p}}{n_{g}}$. In the experiment accurate knowledge of the pulse-to-pulse distance $l_{p p}$ is available since the repetition frequency $f_{r}$ of the laser is locked. Here, $q$ is an integer number and $n_{g}$ is the group refractive index at the peak of the frequency spectrum. We observe that the position where the maximum coherence occurs in the fringe pattern, $d_{m c}$, differs from the position $d_{n_{g}}$. The discrepancy between $d_{m c}$ and $d_{n_{g}}$ is due to the chirp acquired by the pulse, which has an asymmetric spectrum, in the long arm of the interferometer. In Fig. 10 we plot the difference $\left(d_{\text {chirp }}=d_{n_{g}}-d_{m c}\right)$ as a function of the propagation distance in air. Even though, $d_{\text {chirp }}$ is small compared to the delay distance (meters), it has a substantial impact for the evaluation of the delay distance. Our simulations were performed under a fixed environmental condition $\left(20^{\circ} \mathrm{C}\right.$, $1013 \mathrm{hPa}, 45 \%$ humidity). For the numerical integration, a standard resolution of 6000 integration points (corresponding to a $12 \mathrm{~nm}$ step size) and a high resolution of 18000 integration points (corresponding to a $4 \mathrm{~nm}$ step size) were used. This was done mainly to show that the numerical integration has statistical errors when a high precision is required, though these statistical variations can be averaged-out as seen in Fig. 10. The difference between $d_{n_{g}}$ and $d_{m c}$ varies depending on the length scale. The shift of the position of the fringe at maximum coherence of the correlation pattern varies nonlinearly when the delay distance is approximately less than $30 \mathrm{~m}$. After $30 \mathrm{~m}$, this shift stabilizes but always differs from $d_{n_{g}}$.

In Fig. 10 we also plot the $\zeta(X)$ function from Eq. (25) as a function of the delay distance in the interferometer, $X$. We assume that the reference arm placed in air is short $(30 \mathrm{~cm})$, and dispersion in this arm can be neglected. In the long arm, the pulse propagates for several tens of meters. The dispersive term in $\zeta$ is calculated from the equation for refractive index, the Edlén's equation, used previously to derive the distances under the same environmental conditions. It is clearly seen 


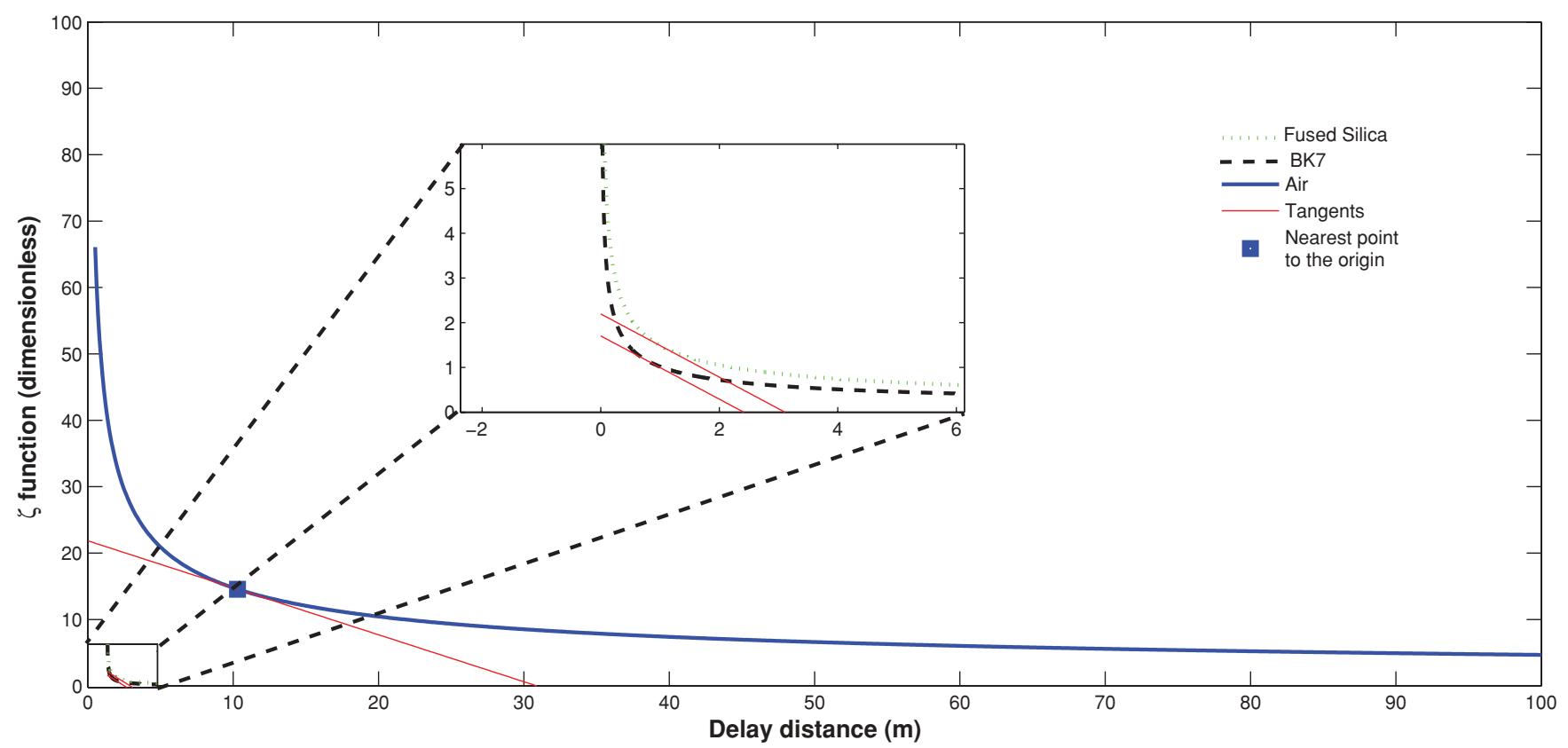

FIG. 11. (Color online) $\zeta$ function showing the nonlinear dispersion depths in various transparent media.

in Fig. 10, that when the propagation distance increases, $\zeta$ tends asymptotically to zero. The asymptotic linear behavior of $\zeta$ starts to dominate the initial nonlinear behavior after approximately $30 \mathrm{~m}$ in air. This property of the function $\zeta$ and the resulting correction $d_{\text {chirp }}$ is dependent on the shape of the PSD of the laser source. If air is the dispersive medium, a variation in the environmental parameters will give rise to a different $\zeta$ function and hence the curve for the correction $d_{\text {chirp }}$ will be different.

\section{NONLINEAR DISPERSION DEPTH IN DISPERSIVE MEDIA}

In the previous section we were able to identify the delay distance in air where nonlinear dispersion effects cease acting on the cross-correlation. Using this method it is possible to indicate the depth of nonlinearity in any optical medium. We do this by choosing a particular point on the $\zeta(X)$ function curve, namely $P\left(X_{p}, Y_{p}\right)$ where $P$ is the nearest point to the origin $O(0,0)$. The distance between the origin and a point $[X, Y=\zeta(X)]$ is defined by $r=\sqrt{X^{2}+\zeta(X)^{2}}$. The point $P$ is located where the smallest value of $r$ occurs. Hence, with $r=r(X)$, the $X_{p}$ coordinate is the solution of the equation $\frac{d}{d X} r(X)=0$. Once $X_{p}$ is obtained, we calculate $Y_{p}$ as $Y_{p}=$ $\zeta\left(X_{p}\right)$. The tangent line $y(X)$ at the point $P\left(X_{p}, Y_{p}\right)$ is given by $y(X)=\zeta^{\prime}\left(X_{p}\right) X+y_{0}$, where $\zeta^{\prime}\left(X_{p}\right)$ is the derivative of $\zeta(X)$ at $X=X_{p}$. Knowing $\zeta^{\prime}\left(X_{p}\right)$ as well as $P\left(X_{p}, Y_{p}\right)$, the intersection point $y_{0}$ of the tangent line at $\left(X_{p}, Y_{p}\right)$ and the $y$-axis can be easily determined.

In Fig. 11 we plot the $\zeta$ function in various media. The tangent lines at the particular points $P$ have also been drawn. We define the distance where nonlinear dispersion is no longer significant as the nonlinear dispersion depth $(\mathcal{D})$. This is the distance after which nonlinear effects do not play a dominant role in the cross-correlation function and linear broadening takes over. This distance $\mathcal{D}$ is obtained as the intersection point of the tangent line with the $\mathrm{x}$-axis. By setting $y=0$, we obtain

$$
\mathcal{D}=-\frac{y_{0}}{\zeta^{\prime}\left(X_{p}\right)}=\frac{3}{2} \sqrt[3]{\frac{\tau_{c}^{2}}{\alpha}} .
$$

The nonlinear dispersion depth is given by Eq. (26) where the light source with a coherence time $\tau_{c}$, a carrier frequency $\omega_{c}$ emits pulses which propagate in a refractive medium with a group delay dispersion $\alpha$ at $\omega_{c}$. For standard air $\mathcal{D}=30 \mathrm{~m}$. Similar calculations and simulations (Fig. 11) are carried out for the $B K 7$ and fused silica glasses. The refractive index equations of these materials have been taken from the Sellmeier equation. We obtained $\mathcal{D}_{B K 7}=2.4 \mathrm{~m}$ and $\mathcal{D}_{\text {silica }}=$ $3 \mathrm{~m}$. The accuracy of the calculated distance or thickness depends on the precision of the refractive index equation. At best, five significant digits are available for Sellmeier's equation, whereas, in the case of the Edlén's equation for air, up to nine significant digits are accurately known. This can be useful for systems using fiber based delay lines.

\section{CONCLUSION}

We have analyzed cross-correlation functions formed after pulse propagation in dispersive media in an unbalanced Michelson interferometer. Discrete models of crosscorrelation patterns generated from the discrete spectrum of the optical frequency comb have been compared to experimental measurements of pulse propagation in $200 \mathrm{~m}$ of air. We observe that the shape of the power spectral density plays a large role in determining the final shape of the correlation pattern. Correlation patterns formed by pulses with symmetric spectra, after propagation in a dispersive media tend to be linearly stretched, leading to an easy retrieval of the position of the brightest fringe, by use of the group refractive index of the medium. In contrast, correlation patterns formed by pulses 
having non symmetric spectra show a shift of the position of the brightest fringe when compared to the position derived from the group refractive index. This shift in the position of the brightest fringe, varies non-linearly with the delay distance and linearly after reaching a certain delay distance. The shift can be predicted using the continuous model for cross-correlation patterns developed in this paper. Using the integral-based continuous cross-correlation function we have demonstrated the behavior of the position of the brightest fringe in the cross-correlation pattern arising from pulses with a nonsymmetric spectra. It has been shown that the delay distance where nonlinearity plays an important role can be scaled for every dispersive medium.

\section{ACKNOWLEDGMENTS}

The authors would like to thank Omar El Gawhary for extensive discussions. The authors gratefully acknowledge J. J. M. Braat for his thoughtful comments on the manuscript. This work is part of the Industrial Partnership Programme (IPP) Metrology with Frequency Comb Laser (MFCL) of the Stichting voor Fundamenteel Onderzoek der Materie (FOM), which is supported financially by Nederlandse Organisatie voor Wetenschappelijk Onderzoek (NWO). The IPP MFCL is co-financed by VSL, TNO, and ASML. The research within this work is also a part of EURAMET joint research project and has received funding from the European Community's Seventh Framework Programme, ERA-NET Plus, under Grant Agreement No. 217257.

\section{APPENDIX}

We begin with the integral representation of the crosscorrelation function

$$
\begin{aligned}
h_{X}(t)= & \frac{1}{\pi} \int_{-\infty}^{\infty} h_{X=0}\left(t_{0}\right) d t_{0} \\
& \times \int_{0}^{\infty} \cos \left[k\left(\omega+\omega_{0}\right) X-\omega \bar{n} \frac{X}{c}+\omega t\right] \cos \left(\omega t_{0}\right) d \omega
\end{aligned}
$$

where $k\left(\omega+\omega_{0}\right)=\alpha \omega^{2}+\beta \omega+\gamma$. Therefore, we obtain

$$
\begin{aligned}
h_{X}(t)= & \frac{1}{\pi} \int_{-\infty}^{\infty} h_{X=0}\left(t_{0}\right) d t_{0} \\
& \times \int_{0}^{\infty} \cos \left[\left(\alpha \omega^{2}+\beta \omega+\gamma\right) X-\omega \bar{n} \frac{X}{c}+\omega t\right] \\
& \times \cos \left(\omega t_{0}\right) d \omega .
\end{aligned}
$$

By converting the product of cosines into a sum of cosines and by completing the square in the cosines of Eq. (A2), we get

$$
\begin{aligned}
h_{X}(t)= & \frac{1}{2 \pi} \int_{-\infty}^{\infty} h_{X=0}\left(t_{0}\right) d t_{0} \int_{0}^{\infty}\left\{\cos \left[\left(\sqrt{\alpha X} \omega-\frac{\left(\beta X-\bar{n} \frac{X}{c}+t+t_{0}\right)}{2 \sqrt{\alpha X}}\right)^{2}+\gamma X-\frac{\left(\beta X-\bar{n} \frac{X}{c}+t+t_{0}\right)^{2}}{4 \alpha}\right]\right. \\
& \left.+\cos \left[\left(\sqrt{\alpha X} \omega-\frac{\left(\beta X-\bar{n} \frac{X}{c}+t-t_{0}\right)}{2 \sqrt{\alpha X}}\right)^{2}+\gamma X-\frac{\left(\beta X-\bar{n} \frac{X}{c}+t-t_{0}\right)^{2}}{4 \alpha X}\right]\right\} d \omega .
\end{aligned}
$$

Next we introduce the following change of variables:

$$
\begin{gathered}
\Omega_{ \pm}=\sqrt{\alpha X} \omega-\frac{\left(\beta X-\bar{n} \frac{X}{c}+t \pm t_{0}\right)}{2 \sqrt{\alpha X}} \\
d \Omega_{ \pm}=\sqrt{\alpha X} d \omega
\end{gathered}
$$

$$
\begin{gathered}
\rho_{ \pm}=\gamma X-\frac{\left(\beta X-\bar{n} \frac{X}{c}+t \pm t_{0}\right)^{2}}{4 \alpha X} \\
\text { and } f_{X}\left(t \pm t_{0}\right)=-\frac{\left(\beta X-\bar{n} \frac{X}{c}+t \pm t_{0}\right)}{2 \sqrt{\alpha X}} .
\end{gathered}
$$

Equation (A2) then simplifies to

$$
\begin{aligned}
h_{X}(t)= & \frac{1}{2 \pi} \int_{-\infty}^{\infty} h_{X=0}\left(t_{0}\right) d t_{0} \int_{f_{X}\left(t \pm t_{0}\right)}^{\infty}\left[\cos \left(\Omega_{+}^{2}+\rho_{+}\right) d \Omega+\cos \left(\Omega_{-}^{2}+\rho_{-}\right) d \Omega\right] \\
= & \frac{1}{4 \pi} \sqrt{\frac{\pi}{2 \alpha X}} \int_{-\infty}^{\infty} h_{X=0}\left(t_{0}\right)\left\{\cos \left(\rho_{+}\right)-2 \cos \left(\rho_{+}\right) \mathcal{C}\left(\sqrt{\frac{2}{\pi}} f_{X}\left(t+t_{0}\right)\right)\right. \\
& -\left[\sin \left(\rho_{+}\right)-2 \sin \left(\rho_{+}\right) \mathcal{S}\left(\sqrt{\frac{2}{\pi}} f_{X}\left(t+t_{0}\right)\right)\right]+\cos \left(\rho_{-}\right)-2 \cos \left(\rho_{-}\right) \mathcal{C}\left(\sqrt{\frac{2}{\pi}} f_{X}\left(t-t_{0}\right)\right) \\
& \left.-\left[\sin \left(\rho_{-}\right)-2 \sin \left(\rho_{-}\right) \mathcal{S}\left(\sqrt{\frac{2}{\pi}} f_{X}\left(t-t_{0}\right)\right)\right]\right\} d t_{0},
\end{aligned}
$$


where the functions $\mathcal{C}$ and $\mathcal{S}$ are the Fresnel integrals and defined as $\mathcal{C}(z)=\int_{0}^{z} \cos \left(\frac{\pi}{2} \xi^{2}\right) d \xi$ and $\mathcal{S}(z)=\int_{0}^{z} \sin \left(\frac{\pi}{2} \xi^{2}\right) d \xi$.

We now consider the case of large delay distance $X$. To be precise, we assume that $X$ is so large that for all $t_{0}$ in the support $\left[-\tau_{c} / 2,-\tau_{c} / 2\right]$ of $h_{X=0}\left(t_{0}\right)$, we have $\frac{\left|t_{0}\right|}{2 \sqrt{\alpha X}} \ll 1$. In this approximation, we rewrite $\rho_{ \pm}$and $f_{X}\left(t \pm t_{0}\right)$ as

$$
\begin{gathered}
\rho_{ \pm}=\gamma X-\frac{\left(\beta X-\bar{n} \frac{X}{c}+t\right)^{2}}{4 \alpha X} \mp \frac{\left(\beta X-\bar{n} \frac{X}{c}+t\right)}{2 \alpha X} t_{0} \\
\text { and } f_{X}(t)=f_{X}\left(t \pm t_{0}\right)=-\frac{\left(\beta X-\bar{n} \frac{X}{c}+t\right)}{2 \sqrt{\alpha X}} .
\end{gathered}
$$

Using the $\rho_{ \pm}$from Eq. (A6) above and expanding the cosines and sines in Eq. (A5) as a trigonometric sum we observe that the integral

$$
\int_{-\infty}^{\infty} h_{X=0}\left(t_{0}\right) \sin \left(\frac{\left(\beta X-\bar{n} \frac{X}{c}+t\right)}{2 \alpha X} t_{0}\right) d t_{0}=0
$$

because $h_{X=0}\left(t_{0}\right)$ is an even function. Therefore, the only contributing terms arise from

$$
\rho=\gamma X-\frac{\left(\beta X-\bar{n} \frac{X}{c}+t\right)^{2}}{4 \alpha X}+\frac{\left(\beta X-\bar{n} \frac{X}{c}+t\right)}{2 \alpha X} t_{0} .
$$

Equation (A5) can be written as

$$
\begin{aligned}
h_{X}(t)= & \frac{1}{2 \pi} \sqrt{\frac{\pi}{2 \alpha X}} \int_{-\infty}^{\infty} h_{X=0}\left(t_{0}\right) \\
& \times\left\{\left[1-2 \mathcal{C}\left(\sqrt{\frac{2}{\pi}} f_{X}(t)\right)\right] \cos (\rho)\right. \\
& \left.-\left[1-2 \mathcal{S}\left(\sqrt{\frac{2}{\pi}} f_{X}(t)\right)\right] \sin (\rho)\right\} d t_{0} .
\end{aligned}
$$

When $a, b$ are real, we can write $a \cos x-b \sin x=R \cos (x+$ $\theta$ ). In our case $R$ and $\theta$ are given by

$$
\begin{aligned}
R & =\sqrt{a^{2}+b^{2}}=\sqrt{\left[1-2 \mathcal{C}\left(\sqrt{\frac{2}{\pi}} f_{X}(t)\right)\right]^{2}+\left[1-2 \mathcal{S}\left(\sqrt{\frac{2}{\pi}} f_{X}(t)\right)\right]^{2}} \\
\text { and } \tan \theta & =\frac{b}{a}=\frac{1-2 \mathcal{S}\left(\sqrt{\frac{2}{\pi}} f_{X}(t)\right)}{1-2 \mathcal{C}\left(\sqrt{\frac{2}{\pi}} f_{X}(t)\right)} .
\end{aligned}
$$

Using this in Eq. (A9), we obtain

$$
\begin{aligned}
h_{X}(t)= & \frac{R}{2 \pi} \sqrt{\frac{\pi}{2 \alpha X}} \int_{-\infty}^{\infty} h_{X=0}\left(t_{0}\right) \\
& \times \cos \left[\gamma X-\frac{\left(\beta X-\bar{n} \frac{X}{c}+t\right)^{2}}{4 \alpha X}\right. \\
& \left.+\frac{\left(\beta X-\bar{n} \frac{X}{c}+t\right)}{2 \alpha X} t_{0}+\theta\right] d t_{0} .
\end{aligned}
$$

We again expand the cosine as a trigonometric sum and using Eq. (A7) we can write $h_{X}(t)$ in a simple integral form as

$$
\begin{aligned}
h_{X}(t)= & \frac{R}{2 \pi} \sqrt{\frac{\pi}{2 \alpha X}} \cos \left[\gamma X-\frac{\left(\beta X-\bar{n} \frac{X}{c}+t\right)^{2}}{4 \alpha X}+\theta\right] \\
& \left.\times \int_{-\infty}^{\infty} h_{X=0}\left(t_{0}\right)\right] \cos \left[\frac{\beta X-\bar{n} \frac{X}{c}+t}{2 \alpha X} t_{0}\right] d t_{0} .
\end{aligned}
$$

[1] D. J. Jones, S. A. Diddams, J. K. Ranka, A. Stentz, R. S. Windeler, J. L. Hall, and S. T. Cundiff, Science 288, 635 (2000).

[2] R. Holzwarth, Th. Udem, T. W. Hänsch, J. C. Knight, W. J. Wadsworth, and P. St. J. Russell, Phys. Rev. Lett. 85, 2264 (2000).

[3] S. T. Cundiff and J. Ye, Rev. Mod. Phys. 75, 325 (2003).

[4] S. A. Diddams, J. C. Bergquist, S. R. Jefferts, and C. W. Oates, Science 306, 1318 (2004).

[5] Th. Udem, R. Holzwarth, and T. W. Hänsch, Nature (London) 416, 233 (2002).

[6] L. Hollberg et al. IEEE J. Quantum Electron. 37, 1502 (2001).
[7] J. Ye, H. Schnatz, and L. W. Hollberg, IEEE J. Sel. Top. Quantum Electron. 9, 1041 (2003).

[8] B. Lamine, C. Fabre, and N. Treps, Phys. Rev. Lett. 101, 123601 (2008).

[9] N. C. Menicucci, S. T. Flammia, and O. Pfister, Phys. Rev. Lett. 101, 130501 (2008).

[10] S. Schiller, Opt. Lett. 27, 766 (2002).

[11] M. J. Thorpe and J. Ye, Appl. Phys. B 91, 397 (2008).

[12] J. Mandon, G. Guelachvili, and N. Picqué, Nature Photonics 3, 99 (2009).

[13] R. Paschotta, A. Schlatter, S. C. Zeller, H. R. Telle, and U. Keller, Appl. Phys. B 82, 265 (2006). 
[14] R. K. Shelton, S. M. Foreman, L. S. Ma, J. L. Hall, H. C. Kapteyn, M. M. Murnane, M. Notcutt, and J. Ye, Opt. Lett. 27, 312 (2002).

[15] T. M. Fortier, D. J. Jones, J. Ye, S. T. Cundiff, and R. S. Windeler, Opt. Lett. 27, 1436 (2002).

[16] J. Ye, Opt. Lett. 29, 1153 (2004).

[17] J. J. M. Braat (unpublished).

[18] M. Cui, R. N. Schouten, N. Bhattacharya, and S. A. van den Berg, J. Europ. Opt. Soc. Rap. Public. 3, 08003 (2008).

[19] Y. Salvade, N. Schuhler, S. Leveque, and S. Le Floch, Appl. Opt. 47, 2715 (2008), and references therein.

[20] K. N. Joo, Y. Kim, and S. W. Kim, Opt. Express 16, 19799 (2008), and references therein.

[21] P. Balling, P. Křen, P. Mašika, and S. A. van den Berg, Opt. Express 17, 9300 (2009).
[22] M. Cui, M. G. Zeitouny, N. Bhattacharya, S. A. van den Berg, H. P. Urbach, and J. J. M. Braat, Opt. Lett. 34, 1982 (2009).

[23] I. Coddington, W. C. Swann, L. Nenadovic, and N. R. Newbury, Nature Photonics 3, 351 (2009).

[24] A. M. Nugrowati, S. F. Pereira, and A. S. van de Nes, Phys. Rev. A 77, 053810 (2008).

[25] L. Wang, H. X. Zhang, and R. P. Wang, JETP Lett. 84, 425 (2006).

[26] K. E. Oughstun and N. A. Cartwright, J. Mod. Opt. 52, 1089 (2005), and references therein.

[27] S. P. Dijaili, A. Dienes, and J. S. Smith, IEEE J. Quantum Electron. 26, 1158 (1990).

[28] J. Jones, Am. J. Phys. 42, 43 (1974).

[29] K. P. Birch and M. J. Downs, Metrologia 31, 315 (1994).

[30] A. Bartels, D. Heinecke, and S. A. Diddams, Opt. Lett. 33, 1905 (2008). 